\title{
Electrodynamics of the planar model: Its phase diagram, continuum limit, and mass spectrum
}

\author{
D. R. T. Jones \\ Theory Division, C.E.R.N., Geneva, Switzerland \\ John Kogut \\ Department of Physics, University of Illinois at Urbana-Champaign, Urbana, Illinois 61801
}

D. K. Sinclair

Department of Applied Mathematics and Theoretical Physics, University of Cambridge, Cambridge, CB3-9EW, England (Received 19 October 1978)

\begin{abstract}
We consider the lattice version of the Abelian Higgs model in $1+1$ dimensions with the Higgs field constrained to have a constant length. In statistical-mechanics terminology this is the planar Heisenberg model coupled to the electromagnetic field. Both the Euclidean Lagrangian and Minkowski Hamiltonian formulations of this lattice gauge theory are used to study its phase diagram, continuum limit, and mass spectrum. The theory contains two dimensionless coupling constants: $\kappa$, the length of the local spin variable, and $x=\left(2 g^{2} a^{2}\right)^{-1}$, where $g$ is the electrodynamic coupling and $a$ is the lattice spacing. We find that the theory confines charges for all $\kappa$ and fixed, finite $x$. However, in the continuum limit $(a \rightarrow 0)$ the theory loses its confinement property and exhibits the Higgs mechanism for $\kappa^{2}>\kappa_{c}{ }^{2}$. The critical point $\kappa_{c}{ }^{2}$ is estimated to lie between 0.6 and 0.8 . If $\kappa^{2}<\kappa_{c}^{2}$, the continuum limit of the theory retains its confining property and appears to be simply the free electromagnetic field (a confining linear potential).
\end{abstract}

\section{INTRODUCTION}

The lattice version of quantum chromodynamics $(\mathrm{QCD})^{1-3}$ is a formulation of the theory which confines quarks for sufficiently large coupling. It is hoped that the continuum limit of the lattice theory also leads to confinement, but this is a delicate question to study since the renormalization group indicates that the coupling vanishes in this regime. ${ }^{4}$ It is certainly possible that the lattice theory passes to an unconfined phase in the limit, although analyses of non-Abelian theories in $3+1$ dimensions happily suggest the contrary. ${ }^{5}$

In this article we study a simple model field theory whose lattice version confines but whose continuum version does so only for a certain range of bare parameters. We will identify the physical origin of the confinement property and understand why it can be lost in the continuum limit. The theory is one whose lattice version has been studied by a host of authors ${ }^{6}$ : the Abelian Higgs model in $1+1$ dimensions with the Higgs field constrained to have a constant length. The lattice version of the model consists of plane rotors at sites coupled together in a gauge-invariant fashion via an electromagnetic field. We therefore refer to it as the "electrodynamics of the planar model." Since the phase diagram of the planar model has been understood in the last few years, we can borrow methods of analysis and physical intuition from statistical mechanics.

We find that the model has the following features. On the lattice it can be rewritten as an ensemble of vortices of finite size interacting through a short-range potential. These vortices are the same as those of the planar model, the coupling to the electromagnetic field having given them finite sizes and short-ranged interactions. These vortices disorder the system destroying the Higgs mechanism and lead to confinement. We make this point by computing the Wilson loop integral in the Euclidean formulation of the model. The theory contains two dimensionless parameters, $\kappa$ the length of the spin variable, and $x=\left(2 g^{2} a^{2}\right)^{-1}$, where $g$ is the electrodynamic coupling and $a$ is the lattice spacing. The computation of the Wilson loop integral shows that for $\kappa^{2} \gtrsim 1 / \pi$ the vortex gas attains a vanishing density in the continuum limit $(g a-0)$ and the confining potential vanishes there. This indicates that the theory exhibits the Higgs phenomenon in the continuum limit. In fact the theory reduces to that of a free massive vector meson in this limit.

The continuum limit for $\kappa^{2} \lesssim 1 / \pi$ appears to be completely different. In this case the Euclidean formulation of the theory indicates that the vortices do not become dilute as $a \rightarrow 0$. This suggests that the continuum limit of the theory should continue to confine. To study this possibility we turn to the Hamiltonian formulation of the theory and compute the interquark potential and low-lying mass spectrum of the theory in strong-coupling expansions through eighth order in $x$. We find that the interquark potential vanishes as $a \rightarrow 0$ for $\kappa^{2}>\kappa_{c}{ }^{2}$ $\approx 0.6-0.8$ by extrapolating the strong-coupling expansions to the continuum $(x \rightarrow \infty)$ limit. We find 
no evidence for any phase transitions at finite $x$. However, for $\kappa^{2}<\kappa_{c}^{2}$ the series calculations indicate that the interquark potential survives in the continuum limit. The series expansions for the lowest-lying vector and scalar states of the lattice theory also indicate that there is a critical point at $\kappa_{c}{ }^{2} \approx 0.6-0.8$. For $\kappa^{2}$ well above the critical point, the ratio of the masses of the scalar and vector states is found to be about 1.84 from the strong-coupling expansions. This is reasonably close to the value 2 expected in the classical continuum limit. However, for $\kappa^{2}<\kappa_{c}^{2}$ the masses themselves appear to grow large as $a-0$ suggesting that in an exact calculation they become infinitely massive and disappear from the spectrum. In summary, for $\kappa^{2}<\kappa_{c}{ }^{2}$ the continuum limit of the lattice theory confines and has no nontrivial spectrum: it appears to be just the free electromagnetic field. Additional evidence for these results is obtained from a very simple implementation of the renormalization-group idea to the Euclidean formulation of the model.

We also study local states in the theory which in strong coupling consist of a fractional charge of magnitude $\alpha$ at one site, a charge of magnitude $-\alpha$ at a neighboring site, and a fractional flux tube connecting them in accordance with Gauss's law. We argue that the parameter $\alpha$ labels bands in the lattice theory which are simply related to various discrete states of the continuum limit of the theory. The strong-coupling expansions for these bands are studied in detail.

The theory is also briefly studied in a background field generated by half a unit of charge at positive spatial infinity and minus half a unit of charge at negative spatial infinity. For $\kappa^{2}$ sufficiently large its vacuum state is a condensate of half-asymptotic particles. ${ }^{7}$

\section{EUCLIDEAN FORMULATION OF THE ELECTRODYNAMICS OF THE PLANAR MODEL}

As discussed in the Introduction, we will construct a tractable lattice model of quark confinement which has the "pathological" property of passing to an unconfined phase at $a=0$ for a range of values of its bare parameters. We will see that for this range of parameters the lattice theory has field configurations which cause confinement but these configurations disappear in the continuum limit. As discussed by Polyakov, ${ }^{8}$ compact quantum electrodynamics in $2+1$ dimensions has this property. The theory we study here contains matter fields and has a more interesting phase diagram. It is a lattice formulation of the Abelian Higgs model in $1+1$ dimensions,

$$
\mathscr{L}=\left|\left(\partial_{\mu}-i g A_{\mu}\right) \phi\right|^{2}-\frac{1}{4}{F_{\mu \nu}}^{2},
$$

where $\phi^{*} \phi=\kappa^{2}=$ const.

As a first step towards an understanding of this theory delete the electromagnetic field from Eq. (2.1) and write the theory on a Euclidean two-dimensional lattice. The constraint $\phi^{*} \phi=\kappa^{2}$ is solved by writing $\phi$ as $\kappa \exp (i \theta)$. A possible twodimensional lattice action is then

$$
A=J \sum_{\langle i j\rangle} \cos \left(\theta_{i}-\theta_{j}\right),
$$

where $\langle i j\rangle$ denotes a sum over nearest neighbors. Equation (2.2) defines the planar model which is the subject of much interest in statistical mechanics. That the naive continuum limit of Eq. (2.2) is the $A_{\mu}=0$ form of Eq. (2.1) is easily verified by considering slowly varying fields, replacing discrete differences by derivatives, and accounting for factors of the lattice spacing $a$ appropriately.

It is useful to review briefly some facts and history concerning the planar model. Old hightemperature expansion $\mathrm{s}^{9}$ indicated the existence of a continuous phase transition at a critical temperature $T_{c}$. The character of the phase transition was conceptually quite puzzling. The low-temperature phase could not be characterized by a spontaneous magnetization [i.e., $\left.\left\langle\exp \left(i \theta_{i}\right)\right\rangle \neq 0\right]$ because a continuous symmetry cannot be spontaneously broken in a two-dimensional system with shortrange forces. ${ }^{10}$ This puzzle was solved by the work of Kosterlitz and Thouless ${ }^{11}$ who pointed out that there are two distinct types of stationary spin configurations of the action Eq. (2.2). A constantspin field is one case. Another is a vortex configuration shown in Fig. 1. Vortices have several distinctive properties. They are quantized. Their action varies as $\ln (R / a)$ where $R$ is the linear size of the spin system and $a$ is the lattice spacing. To

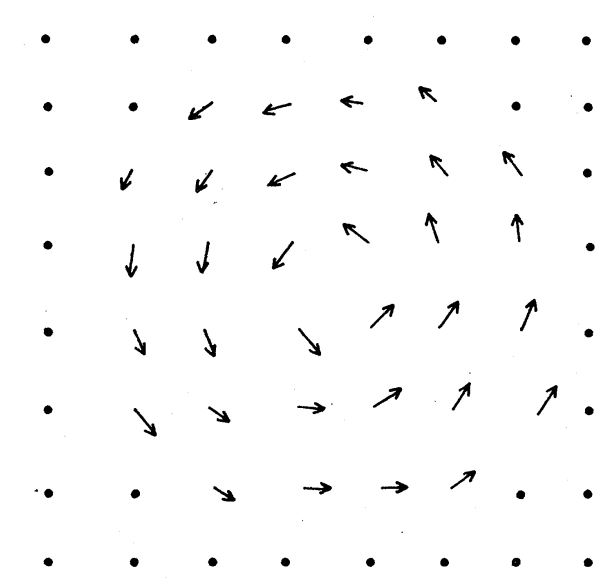

FIG. 1. The spin configuration making up a vortex. 
see this approximate Eq. (2.2) by the continuum expression, $J \sum_{r}[\nabla \theta(r)]^{2}$, and evaluate it for the vortex of Fig. 1,

$$
\text { action (vortex) } \approx \pi J \ln (R / a) \text {. }
$$

The singular character of this spin configuration is reflected in the fact that Eq. (2.3) diverges as $a \rightarrow 0$. As will be discussed further below, the fact that Eq. (2.3) has a large volume divergence means that vortices are important in the model only for sufficiently large temperatures. In fact, Kosterlitz and Thouless ${ }^{11}$ suggested that it is the presence or absence of vortices which distinguish the low - and high-temperature phases of the model. They suggested that below $T_{c}$ the only significant excitations are conventional spin waves, but as $T$ is increased above $T_{c}$ bound pairs of a vortex and an antivortex can dissociate and influence the longdistance behavior of the system. Clearly free vortices tend to disorder the system. A renormalization-group analysis ${ }^{12}$ of the model indicates that below $T_{c}$ the spin-spin correlation functions are power-behaved in the distance between spins while above $T_{c}$, where vortices are relevant, the correlation function falls exponentially. Kosterlitz and Thouless ${ }^{11}$ presented a very informal but instructive determination of $T_{c}$. They argued that free vortices will appear in the system when their contribution to the free energy $F$ becomes negative. The energy $E$ of a free vortex is recorded in Eq. (2.3) and its entropy $S$ is obtained by counting the number of places it can occur on the lattice,

$$
E=\pi J \ln (R / a), \quad S=k \ln (R / a)^{2},
$$

where $k=$ Boltzmann's constant. So,

$$
F=E-T S=(\pi J-2 k T) \ln (R / a)
$$

becomes negative for $T$ greater than $T_{c}=\pi J / 2 k$. It is easy to argue that for $T<T_{c}$ the spin-spin correlation function is power-behaved. To see this suppose $T<T_{c}$ so vortices can be ignored. Replace the action of Eq. (2.2) by its continuum form $J \sum_{r}[\nabla \theta(r)]^{2}$ and extend the range of the variable $\theta$ to $[-\infty, \infty]$. Then,

$$
\left\langle e^{i\left(\theta_{i}-\theta_{j}\right)}\right\rangle=\frac{\int \prod_{i} d \theta_{i} \exp \left[-(J / k T) \sum_{r}(\nabla \theta)^{2}\right] e^{i\left(\theta_{i}-\theta_{j}\right)}}{\int \prod_{i} d \theta_{i} \exp \left[-(J / k T) \sum_{r}(\nabla \theta)^{2}\right]}
$$

which is easily evaluated since all the integrals are Gaussian,

$$
\begin{aligned}
&\left\langle e^{i\left(\theta_{i}-\theta_{j}\right)}\right\rangle=\exp \left[\frac{k T}{2 J} \Delta\left(r_{i}-r_{j}\right)\right] \\
& \underset{\left|r_{i}^{-} r_{j}\right| \gg a}{\sim} \exp \left[-\frac{k T}{4 \pi J} \ln \left|\frac{r_{i}-r_{j}}{r_{0}}\right|\right],
\end{aligned}
$$

where $\Delta\left(r_{i}-r_{j}\right)$ is a massless propagator (on a lattice) and we have used its asymptotic form in the last step. So, finally,

$$
\left\langle e^{i\left(\theta_{i}-\theta_{j}\right)}\right\rangle \sim\left|\frac{r_{0}}{r_{i}-r_{j}}\right|^{k T / 4 \pi J},
$$

for $T<T_{c}$. We learn from this that spin waves cause the correlation function to be power-behaved so $\left\langle e^{i \theta_{i}}\right\rangle=0$ and there is no spontaneous magnetization at any $T$. A renormalization-group analysis ${ }^{12}$ shows that the line of fixed points in Eq. (2.7) terminates into a continuous phase transition at $T_{c}$ above which the correlation function becomes an exponential in $\left(r_{i}-r_{j}\right)$.

Now return to Eq. (2.1). This theory is trivially soluble. Make a gauge transformation,

$$
D_{\mu}=A_{\mu}+\frac{1}{g} \partial_{\mu} \theta
$$

where $\theta$ is defined via the relation,

$$
\phi=\kappa e^{i \theta}, \quad \kappa=\text { positive constant . }
$$

Substituting into Eq. (2.1) gives

$$
\mathcal{L}=-\frac{1}{4} F_{\mu \nu}{ }^{2}+g^{2} \kappa^{2} D_{\mu}{ }^{2},
$$

which describes a free vector meson of mass

$$
m_{V}=\sqrt{2} g \kappa .
$$

This theory does not, of course, confine. If we couple charged static sources to the field $A_{\mu}$, and then imagine slowly separating a pair of such impurities (of charge $+e$ and $-e$, say), the energy required would be finite since the gauge field $D_{\mu}$ is free and massive.

This analysis does not, of course, mean that the continuum limit of a lattice formulation of this model will be Eq. (2.10). For example, the lattice planar model becomes a massless free field in the continuum limit only for sufficiently low temperatures. This fact suggests that the continuum limit of the electrodynamics of the planar model will be Eq. (2.10) only for $\kappa$ sufficiently large. The analysis presented in this paper will support this view and will make it precise.

Now we shall construct the lattice version of this theory and derive its confining properties. Before beginning a detailed analysis we can anticipate many of the results using the facts concerning the planar model reviewed above. The lattice version of Eq. (2.1) will contain both spin waves and vortices. But, since Eq. (2.1) contains a dimensionful parameter $g$, or equivalently the mass $m_{V}$, the energetics of the theory are different from the planar model. The parameter $m_{V}$ will act as an infrared cutoff so the action of a vortex will vary as $\ln \left(m_{V} a\right)$. So, for finite $m_{V} a$, vortices will be important excitations. Their presence will 
lead to confinement. For $\kappa^{2}$ sufficiently large confinement will be lost precisely in the continuum limit since the action of a vortex diverges at this point.

The lattice version of Eq. (2.1) is obtained following the original ideas of Wilson ${ }^{2}$ and Polyakov ${ }^{1}$ on compact $Q E D$. Define the vector variable $B_{\mu}(r)=a g A_{\mu}(r)$ on the link connecting the sites $r$ and $r+\hat{\mu}$ on a square Euclidean two-dimensional lattice labeled by integers $r=\left(r_{1}, r_{2}\right) . \Delta_{\mu}$ will denote a discrete difference operator,

$$
\Delta_{\mu} f(r)=f(r+\hat{\mu})-f(r) .
$$

The lattice action replacing Eq. (2.1) is then

$$
\begin{aligned}
A= & 2 \kappa^{2} \sum_{r, \mu} \cos \left[\Delta_{\mu} \theta(r)+B_{\mu}(r)\right] \\
& +\frac{1}{2 g^{2} a^{2}} \sum_{r, \mu, \nu} \cos \left[\Delta_{\mu} B_{\nu}(r)-\Delta_{\nu} B_{\mu}(r)\right] .
\end{aligned}
$$

Some of the important properties of $A$ are the following. It is locally gauge invariant, i.e., the lattice gauge transformation,

$B_{\mu}(r)-B_{\mu}(r)+\Delta_{\mu} \Lambda(r), \quad \theta(r)-\theta(r)-\Lambda(r)$

leaves $A$ invariant. $B_{\mu}(r)$ is effectively an angular variable so in the partition function it will be integrated from 0 to $2 \pi . \theta(r)$ is also an angular variable.

To discuss the confining properties of this theory we need to construct a suitable correlation function. This is the Wilson loop integral,

$$
\left\langle\exp \left(i e \int_{C} A_{\mu} d x^{\mu}\right)\right\rangle=\frac{\int \prod_{r} d \theta(r) \prod_{r^{\prime}, \mu} d A_{\mu}\left(r^{\prime}\right) \exp \left(-A+i e \int A_{\mu} d x^{\mu}\right)}{\int \prod_{r} d \theta(r) \prod_{r^{\prime}, \mu} d A_{\mu}\left(r^{\prime}\right) e^{-A}}
$$

where $C$ is a closed contour. Equation (2.14) gives the action required to move a static charge $e$ around the contour $\dot{C}$. It is convenient to consider a contour $C$ such as the one in Fig. 2 having $T \gg R$. In this case heavy quarks of charge $+e$ and $-e$ are adiabatically separated a distance $R$, are then held there for $T \gg R$, and are then brought back together. The energy required to separate the quarks a distance $R$ is then obtained from the Wilson line integral through the relation

$$
E(R)=-\lim _{T \rightarrow \infty} \frac{1}{T} \ln \left\langle\exp \left(i e \int A_{\mu} d x^{\mu}\right)\right\rangle
$$

and $E(R)$ can be used to label the phases of the theory. If $E(R) \rightarrow 0$ as $R \rightarrow \infty$, the theory is in the unconfined phase. If $E(R) \sim R$ as $R \rightarrow \infty$ for $e$ different than an integral multiple of $g$ and if $E(R)$ $\rightarrow 0$ as $R \rightarrow \infty$ for $e$ equal to an integral multiple of $g$, then the theory is in the confining phase. These are the standard definitions and the reader should consult the literature for further discussion. ${ }^{13}$

Next we must write $\int A_{\mu} d x^{\mu}$ using lattice variables. Introduce the vector field $J_{\mu}(r)$ to label the contour $C$ :

$J_{\mu}(r)=\left\{\begin{array}{l}+1 \text { if the link } r-r+\mu \text { is on the contour } C, \\ -1 \text { if the link } r+\mu \rightarrow r \text { is on the contour } C, \\ 0 \text { otherwise. }\end{array}\right.$
Using the definition $B_{\mu}=a g A_{\mu}$ we have the correspondence,

$$
e \int A_{\mu} d x^{\mu}-\frac{e}{g} \sum_{c} B_{\mu} J_{\mu} .
$$

Finally, if we define

$$
\begin{aligned}
Z(J)=\int \prod_{r} d \theta(r) \prod_{r^{\prime}, \mu} & d A_{\mu}\left(r^{\prime}\right) \\
& \times \exp \left(-A-i \frac{e}{g} \sum_{c} B_{\mu} J_{\mu}\right),
\end{aligned}
$$

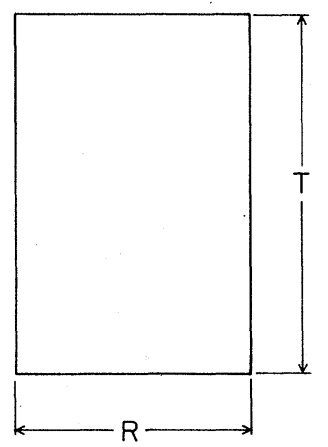

FIG. 2. The contour $C$ chosen to compute the interquark potential. 
then Wilson's correlation function is $Z(J) / Z(0)$.

Our next task is the evaluation of Eq. (2.17).

This will be done using the Villain approximation. ${ }^{14}$ To motivate the following we concede that we can only evaluate Gaussian functional integrals in closed form. However, the action $A$ depends on angular variables $\theta$ and $B_{\mu}$ and we must preserve this property of the model in any approximation procedure. Villain invented the periodic Gaussian approximation for just this situation. He noted that for large $\beta$,

$$
e^{-\beta(1-\cos \theta)} \approx \frac{1}{\sqrt{2 \pi \beta}} \sum_{n=-\infty}^{\infty} e^{i n \theta} e^{-n^{2} / 2 \beta},
$$

which is just the Frouier series analysis of the periodic function $\exp [-\beta(1-\cos \theta)]$. We will make the following replacements in the action Eq. (2.12),

$$
\begin{gathered}
e^{-2 \kappa^{2}\left[1-\cos \left(\Delta_{\mu} \theta-B_{\mu}\right)\right]}-\sum_{n_{\mu}=-\infty}^{\infty} e^{i n_{\mu}\left(\Delta_{\mu} \theta-B_{\mu}\right)} e^{-n^{2} / 4 \kappa^{2}}, \\
\exp \left(-\frac{1}{2 g^{2} a^{2}}\left[1-\cos \left(\Delta_{\mu} B_{\nu}-\Delta_{\nu} B_{\mu}\right)\right]\right) \\
-\sum_{l_{\mu \nu}=-\infty}^{\infty} e^{i l_{\mu \nu}\left(\Delta_{\mu} B_{\nu}-\Delta_{\nu} B_{\mu}\right)} e^{-g^{2} a^{2} l_{\mu \nu}{ }^{2} / 4} .
\end{gathered}
$$

These replacements preserve the periodicity of the variables $\theta$ and $B_{\mu}$, the local gauge invariance and the continuum limit. Therefore, they serve as another definition of the lattice theory for all values $\kappa^{2}$ and $g^{2} a^{2}$ of the model. Since the Villain form of the theory is tractable we concentrate on it from here on. In addition, to preserve periodicity in the presence of an external current loop; we should give the matter field a charge $p$ and consider external charges $q=0,1, \ldots, p$. This point will be left implicit below.

Using the replacements Eq. (2.18) in the partition function $Z(J)$ we can then do the functional integrations over $\theta(r)$ and $B_{\mu}(r)$,

$$
\begin{aligned}
Z(J)= & \prod_{r, \mu, \nu} \sum_{l_{\mu \nu}(r)} \prod_{r^{\prime}, \mu^{\prime}} \delta_{\Delta^{\nu} l_{\mu \nu}\left(r^{\prime}\right)+(e / g) J_{\mu}\left(r^{\prime}\right)+n_{\mu}\left(r^{\prime}\right), 0} \\
& \times \prod_{r^{\prime \prime}} \sum_{n_{\mu}\left(r^{\prime \prime}\right)} \prod_{r^{\prime \prime \prime}} \delta_{\Delta_{\mu} n_{\mu}\left(r^{\prime \prime \prime}\right), 0} \\
& \times e^{-n_{\mu}{ }^{2}\left(r^{\prime \prime}\right) / 4 \kappa^{2}} e^{-g^{2} a^{2} l_{\mu \nu}{ }^{2}(r) / 4}
\end{aligned}
$$

These integrals have generated constrained sums over integer-valued fields. This phenomenon has been noted and studied recently by several authors. ${ }^{15,16}$ It is best to solve the constraints explicitly. Since the fields $n_{\mu}$ and $J_{\mu}$ are divergence free, they can be written as curls of other integer- valued fields,

$$
n_{\mu}=\epsilon_{\mu \nu} \Delta_{\nu} n, \quad J_{\mu}=\epsilon_{\mu \nu} \Delta_{\nu} J
$$

Finally, the constraint

$$
\Delta_{\nu} l_{\mu \nu}+\frac{e}{g} J_{\mu}+n_{\mu}=0
$$

can be solved,

$$
\begin{aligned}
l_{\mu \nu} & =m^{\mu}(m \cdot \Delta)^{-1}\left(\frac{e}{g} J^{\nu}+n^{\nu}\right) \\
& -m^{\nu}(m \cdot \Delta)^{-1}\left(\frac{e}{g} J^{\mu}+n^{\mu}\right),
\end{aligned}
$$

where the expression $(m \cdot \Delta)^{-1}$ means a discrete line integral along the direction $m$,

$$
(m \cdot \Delta)^{-1} f\left(r_{1}, r_{2}\right)=\sum_{m^{\prime}=-\infty}^{r_{2}} f\left(r_{1}, m^{\prime}\right) .
$$

We choose $m$ in the two direction for convenience. This choice will soon disappear from the mathematics. Next we can write the Gaussian functions appearing in Eq. (2.19) in terms of $n$, and $J$,

$$
\begin{aligned}
& n_{\mu} n_{\mu}=\left(\Delta_{\mu} n\right)^{2}, \\
& l_{\mu \nu} l_{\mu \nu}=2\left(\frac{e}{g} J+n\right)^{2} .
\end{aligned}
$$

So, now $Z(J)$ has the simpler form,

$$
\begin{aligned}
Z(J)=\sum_{n(r)=-\infty}^{\infty} \exp [ & -\frac{1}{4 \kappa^{2}} \sum_{r}\left[\Delta_{\mu} n(r)\right]^{2} \\
& \left.-\frac{g^{2} a^{2}}{2} \sum_{r}\left(n(r)+\frac{e}{g} J(r)\right)^{2}\right] .
\end{aligned}
$$

If $g^{2}$ were zero, we could recognize this expression as the interface-roughening model. ${ }^{17}$ Equation (2.24) is a convenient expression in our case only when $g^{2} a^{2}$ is large so that the sum over $n(r)$ is quickly convergent. We will return to Eq. (2.24) below, but now we will write $Z(J)$ in a convenient form for small $g^{2} a^{2}$. To do this we borrow another trick from the recent studies of the planar mod$\mathrm{el}^{16}$ - the Poisson summation formula which allows us to rewrite the sum over integers in a different way,

$$
\sum_{l=-\infty}^{\infty} h(l)=\sum_{m=-\infty}^{\infty} \int_{-\infty}^{\infty} d \phi h(\phi) e^{2 \pi i m \phi},
$$

where $h$ is arbitrary. Applying this trick to Eq. (2.24), 


$$
Z(J)=\int_{-\infty}^{\infty} \prod_{r} d \phi(r) \sum_{m(r)_{z}=\infty}^{\infty} \exp \left[-\frac{1}{4 \kappa^{2}} \sum_{r}\left(\Delta_{\mu} \phi\right)^{2}-\frac{g^{2} a^{2}}{2} \sum_{r}\left(\phi+\frac{e}{g} J\right)^{2}+2 \pi i \sum_{r} m(r) \phi(r)\right] .
$$

Doing the integrations over $\phi(r)$ and dropping an overall constant we have

$$
\begin{aligned}
Z(J)=\sum_{m(r)=-\infty}^{\infty} \exp ( & -4 \pi^{2} \kappa^{2} \sum_{r_{\bullet} r^{\prime}} m(r) G\left(r-r^{\prime} ; m_{V} a\right) m\left(r^{\prime}\right)-4 \kappa^{2} \pi i \frac{e}{g}\left(g^{2} a^{2}\right) \sum_{r_{\bullet} r^{\prime}} m(r) G\left(r-r^{\prime} ; m_{V} a\right) J\left(r^{\prime}\right) \\
+ & \left.4 \kappa^{2}\left(g^{2} a^{2}\right)^{2} \frac{e^{2}}{g^{2}} \sum_{r_{\bullet} r^{\prime}} J(r) G\left(r-r^{\prime} ; m_{V^{\prime}} a\right) J\left(r^{\prime}\right)-\frac{1}{2} g^{2} a^{2} \frac{e^{2}}{g^{2}} \sum_{r} J(r)^{2}\right),
\end{aligned}
$$

where $G$ is the lattice propagator coming from inverting the lattice form of the operator $\left(\vec{\nabla}^{2}\right.$ $\left.+m_{V}^{2} a^{2}\right)$

$G\left(r ; m_{V} a\right)=\int_{-\pi}^{\pi} \frac{d k_{x}}{2 \pi} \int_{-\pi}^{\pi} \frac{d k_{y}}{2 \pi} \frac{e^{i \overrightarrow{\mathrm{x}} \cdot \overrightarrow{\mathrm{r}}}}{4-2 \cos k_{x}-2 \cos k_{y}+m_{V}{ }^{2} a^{2}}$.

To expose the physics in Eq. (2.27) consider the case $J=0$,

$$
\begin{aligned}
Z(J=0)=\sum_{m(r)=-\infty}^{\infty} \exp \left(-4 \pi^{2} \kappa^{2} \sum_{r, r^{\prime}}\right. & m(r) \\
& \left.\times G\left(r-r^{\prime} ; m_{V} a\right) m\left(r^{\prime}\right)\right),
\end{aligned}
$$

which expresses the partition function as integervalued fields $m(r)$ interacting through short-range potentials $G\left(r, m_{V} a\right)$. As will become clearer below $m(r)$ represent the vortices which are interacting through the field identified in Eq. (2.10) - the massive, free vector field. Note that if $g=0$ here $Z(0)$ reduces to the two-dimensional Coulomb gas. ${ }^{16}$

Equation (2.27) is a convenient form of the partition function for small $a$. We will now evaluate it for a suitable contour $(T \gg R)$ and verify that for $\kappa$ sufficiently large the theory confines except at $a=0$. This analysis will be easy for two reasons. First, the self-energy of a vortex diverges as $a \rightarrow 0$, so their density on the lattice will be small. In addition, they interact through shortrange forces, so for " $a$ " small they can be approximated by a free, noninteracting gas. The term $r=r^{\prime}$ in the sum of Eq. (2.29) gives the selfenergy

$$
G\left(0, m_{V} a\right) \sim-\frac{1}{2 \pi} \ln \left(m_{V} a\right),
$$

so neglecting interactions between vortices,

$$
Z(0) \approx \sum_{m(r)} \exp \left(2 \pi \kappa^{2} \ln (\sqrt{2} \kappa g a) \sum_{r} m(r)^{2}\right) .
$$

The interactions between vortices can be ignored if they usually lie outside each other's range of influence which is $\sim\left(m_{V} a\right)^{-1}$. But Eq. (2.31) implies that the probability to find a vortex on a given lattice site is $\left(m_{V} a\right)^{2 \pi k^{2}}$. So, the probability that a second vortex lies close enough to interact with a given one is roughly

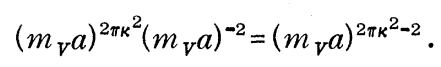

So, if $\kappa^{2}>1 / \pi$ this quantity vanishes as $a \rightarrow 0$ and Eq. (2.31) is a sensible approximation to $Z(J=0)$. If $\kappa$ violates this inequality the dilute-gas approximation fails and other methods of analysis (an attractive possibility is a theory of screening implemented via a renormalization group) are necessary. Strong-coupling expansions will be used in the next section of this paper to investigate the theory for arbitrary $\kappa^{2}$.

The analysis of Eq. (2.27) is now straightforward and bears a strong resemblance to the instanton calculations of Ref. 18. First note that for the contour of Fig. 2, $J(r)=+1$ if $r$ is inside the contour and zero otherwise. Then $J_{\mu}=\epsilon_{\mu \nu} \Delta_{\nu} J$ is +1 on the left vertical piece of $C$ and -1 on the right vertical piece. Aside from an overall factor of $g^{2} a^{2}\left(e^{2} / g^{2}\right)$ the last two terms in Eq. (2.27) become

$$
\left(m_{V} a\right)^{2} \sum_{\substack{r, r^{\prime} \\ \text { inside }}} G\left(r-r^{\prime} ; m_{V} a\right)-R T
$$

But for $R$ and $T \gg\left(m_{V} a\right)^{-1}$, the dominant piece of the double sum in Eq. (2.33) is easily obtained. Fix $r^{\prime}$ inside and not near the contour. Then the sum over $r$ can be done because then the extremities in the sum can be ignored. But

$$
\sum_{r} G\left(r ; m_{V} a\right)=\frac{1}{\left(m_{V} a\right)^{2}}
$$

so Eq. (2.33) becomes 


$$
\begin{aligned}
\left(m_{V} a\right)^{2}\left(\sum_{\substack{r^{\prime} \\
\text { inside }}}\right)\left(\sum_{r} G\left(r ; m_{V} a\right)\right)-R T \\
\quad=\left(m_{V} a\right)^{2} \frac{1}{\left(m_{V} a\right)^{2}} R T-R T=0
\end{aligned}
$$

to leading order in $R T$. Similar approximations give

$$
\sum_{r, r^{\prime}} m(r) G\left(r-r^{\prime} ; m_{V} a\right) J\left(r^{\prime}\right) \approx \frac{1}{m_{V}^{2}} \sum_{r}^{\text {inside }} m(r) \text {. }
$$

So, finally we have a simple expression for $Z(J)$,

$$
\begin{gathered}
Z(J)=\sum_{m(r)} \exp \left(2 \kappa^{2} \pi \ln (\sqrt{2} \kappa g a) \sum_{r} m^{2}(r)\right. \\
-2 \pi i \frac{e}{g} \sum_{r}^{\text {inside } m(r)) .}
\end{gathered}
$$

Since we are taking $a$ to be small the sum over vortices can be truncated after $m(r)=0, \pm 1$. Then if $r$ is inside the contour,

$$
\begin{aligned}
\sum_{m(r)} \exp \left(2 \pi \kappa^{2} \ln \left(m_{V} a\right) m(r)^{2}-2 \pi i \frac{e}{g} m(r)\right) \\
\approx 1+2 \exp \left[2 \pi \kappa^{2} \ln \left(m_{V} a\right)\right] \cos \left(2 \pi \frac{e}{g}\right) \\
\approx \exp \left[2 e^{2 \pi \kappa^{2} \ln \left(m_{V} a\right)} \cos \left(2 \pi \frac{e}{g}\right)\right] .
\end{aligned}
$$

So, we obtain for the Wilson loop integral,

$$
\frac{Z(J)}{Z(0)} \approx \exp \left\{2\left(m_{V} a\right)^{2 \pi k^{2}} \sum_{r}^{\text {ins } 1 \mathrm{de}}\left[\cos \left(2 \pi \frac{e}{g}\right)-1\right]\right\} \text {. }
$$

So,

$$
\frac{Z(J)}{Z(0)} \cong \exp \left[-2\left(m_{V} a\right)^{2 \pi \kappa^{2}}\left(1-\cos 2 \pi \frac{e}{g}\right) R T\right],
$$

from which we read off $E(R)$ from the prescription in Eq. (2.15)

$$
E(R)=2 m_{V}^{2}\left(m_{V} a\right)^{2 \pi \kappa^{2}-2}\left(1-\cos 2 \pi \frac{e}{g}\right) R,
$$

where we have restored physical units (i.e., $R$ could be in $\mathrm{GeV}^{-1}$ units).

We read off several facts from Eq. (2.41). The coefficient of the linear force law is periodic in $e / g$. If $e / g$ is an integer it vanishes. Thus, we have the standard picture of confinement. Note that the coefficient of $E(R)$ vanishes in the continuum limit for $\kappa^{2}>1 / \pi$ and confinement is lost precisely at $a=0$.

Finally, let us briefly consider confinement for large $a$. Then it is best to return to Eq. (2.24) for $Z(J)$,

$$
\begin{aligned}
Z(J)=\sum_{n(r)=-\infty}^{\infty} \exp [ & -\frac{1}{4 \kappa^{2}} \sum_{r}\left(\Delta_{\mu} n\right)^{2} \\
& \left.-\frac{g^{2} a^{2}}{2} \sum_{r}\left(n(r)+\frac{e}{g} J(r)\right)^{2}\right] .
\end{aligned}
$$

The absence of a long-range force in the case $e / g$ = integer is obvious from this expression-simply translate the sum over $n(r)$ and the factor $(e / g) J(r)$ disappears except for some short-range effects. Now suppose $e / g \neq$ integer. Then the most important configuration $\{n(r)\}$ will be

$$
\text { inside } C: n(r)=\text { nearest integer to }-\frac{e}{g},
$$$$
\text { outside } C: n(r)=0 \text {. }
$$

Suppose $|e / g|$ is much smaller than unity, so the dominant configuration $\{n(r)\}$ is zero everywhere. Aside from some short-range terms, the Wilson loop integral becomes

$$
Z(J) / Z(0) \approx e^{-e^{2} a^{2} R T / 2},
$$

so that

$$
E(R)=\frac{1}{2} e^{2} R
$$

in physical units. Recognize this result as characteristic of an electric flux tube, completely uneffected by the vacuum, extending between the impurities $\pm e$. The behavior of $E(R)$ for larger $|e|$ is now clear: $E(R)$ rises as $e^{2}$ until $|e|=\frac{1}{2}|g|$ where its slope changes sign. It vanishes at $|e|$ $=|g|$. In general, $E(R)$ is a periodic function of $|e|$ with period $|g|$.

In summary now, we see that this theory displays confinement both for strong coupling (large $g^{2} a^{2}$ ) and for weak coupling ( $\mathrm{small} \mathrm{g}^{2} a^{2}$ ) and loses this property precisely at $a^{2}=0$. Although we have not analyzed the intermediate coupling regime in detail, there is no reason to expect a transition separating weak and strong coupling. We could, of course, use the interface roughening form of the theory, Eq. (2.24), and develop a strong-coupling expansion for the string tension. Instead, we turn to the Hamiltonian form of the theory and use strong coupling and Padé approximants to analyze the same physics for all values of $\kappa^{2}$ and $x$.

\section{HAMILTONIAN FORMULATION}

In order to study the theory in more detail we return to real space-time and consider the Hamiltonian formulation of the theory on a one-dimensional spatial lattice, with time kept continuous. ${ }^{3}$ We choose that class of gauges for which $A_{0}=0$. For the Lagrangian of Eq. (2.1) where $\phi^{\dagger} \phi=\kappa^{2}$, our momentum conjugate to $\theta\left(\phi \equiv \kappa e^{i \theta}\right)$ is 


$$
\pi=2 \kappa^{2} \partial_{0} \theta
$$

and the Hamiltonian density

$$
\begin{aligned}
\mathcal{H} C & =\frac{1}{4 \kappa^{2}} \pi^{2}+\kappa^{2}\left(\partial_{1} \theta\right)^{2}+2 \kappa^{2} g\left(\partial_{1} \theta\right) A_{1}+\kappa^{2} g^{2} A_{1}{ }^{2}+\frac{1}{2} E^{2} \\
& =\frac{1}{4 \kappa^{2}} \pi^{2}+\partial_{1} \phi^{\dagger} \partial_{1} \phi-i g \phi^{\dagger} \ddot{\partial}_{1} \phi A_{1}+\kappa^{2} g^{2} A_{1}{ }^{2}+\frac{1}{2} E^{2} .
\end{aligned}
$$

Now consider the transition to the lattice of spacing $a$. The equal-time commutation relation

$$
[\pi(x), \theta(y)]=-i \delta(x-y)
$$

becomes

$$
[\pi(n), \theta(m)]=-i \delta_{m n},
$$

where

$$
\begin{aligned}
& \pi(n) \equiv a \pi(n a), \\
& \theta(n) \equiv \theta(n a),
\end{aligned}
$$

with $n$ an integer. In terms of $\phi(n)=\kappa e^{i \theta(n)} \mathrm{Eq}$.

(3.3) becomes

$$
\begin{aligned}
& {[\pi(n), \phi(m)]=-\delta_{m n} \phi(n),} \\
& {\left[\pi(n), \phi^{\dagger}(m)\right]=\delta_{m n} \phi^{\dagger}(m) .}
\end{aligned}
$$

Following the technique used for $(1+1)$-dimensional QED ${ }^{19}$ and both $(1+1)$ - and $(3+1)$-dimensional $\mathrm{QCD},{ }^{3}$ we assign our electromagnetic field to the links of the lattice and define

$$
U(n, n+1)=\exp \left[\operatorname{iga} A_{1}(n)\right] .
$$

Our gauge-invariant Hamiltonian $H=\int d x \mathcal{H}(x)$ then becomes

$$
\begin{aligned}
H=\frac{1}{2} \sum_{n} a g^{2} L^{2}(n)+\sum_{n} \frac{1}{2 a \kappa^{2}} \pi^{2}(n) \\
-\frac{1}{a} \sum_{n}\left[\phi^{\dagger}(n) U(n, n+1) \phi(n+1)\right. \\
\left.+\phi^{\dagger}(n+1) U^{\dagger}(n, n+1) \phi(n)-2 \kappa^{2}\right],
\end{aligned}
$$

on the lattice where

$$
L(n)=\frac{1}{g} E(n a) .
$$

Since $E$ and $A_{1}$ are canonically conjugate, the lattice variables $L(n)$ and $a g A_{1}(n)$ are canonically conjugate,

$$
\left[L(n), a g A_{1}(m)\right]=\delta_{n, m} .
$$

For the purpose of setting up the lattice perturbation theory, we define

$$
\begin{aligned}
W=\frac{2}{a g^{2}} H=\sum_{n}\{ & L^{2}(n)+\frac{x}{\kappa^{2}} \pi^{2}(n) \\
& -4 x\left[\phi^{\dagger}(n) U(n, n+1) \phi(n+1)\right. \\
& \left.\left.+ \text { H.c. }-2 \kappa^{2}\right]\right\},
\end{aligned}
$$

where $x=1 /\left(2 a^{2} g^{2}\right)$. We can now formulate perturbation theory about $a=\infty$, i.e., $x=0$ and attempt to continue series expansions to $a=0$, i.e., $x=\infty$, by use of Padé approximants.

It will often be found useful to consider the simpler Hamiltonian obtained from the above when $\pi^{2}(n)=0$, i.e., $\phi$ is treated classically (unquantized). In this approximation the modes for each link decouple and the theory is exactly soluble. The Hamiltonian is (up to an overall factor of 2/ $a g^{2}$ )

$$
\begin{gathered}
W^{\prime}=\sum_{n}\left\{L^{2}(n)-4 x\left[\phi^{\dagger}(n) U(n, n+1) \phi(n+1)\right.\right. \\
\left.\left.+ \text { H.c. }-2 \kappa^{2}\right]\right\} .
\end{gathered}
$$

A simple gauge transformation reduces all the $\phi(n)$ 's to $\kappa$ giving

$$
\begin{aligned}
W^{\prime}=\sum_{n}\left\{L^{2}(n)-4 x \kappa^{2}[\right. & U(n, n+1) \\
& \left.\left.+U^{\dagger}(n, n+1)-2\right]\right\} .
\end{aligned}
$$

As promised, the Hamiltonians for each link are decoupled. For any link the Hamiltonian is of the form

$$
W_{1 \mathrm{ink}}=L^{2}-4 x \kappa^{2}\left(U+U^{\dagger}-2\right) .
$$

If we set $U=e^{i \times}$ then on the space of functions of $\chi$,

$$
W_{1 \mathrm{ink}}=-\frac{d^{2}}{d \chi^{2}}-8 x \kappa^{2}(\cos \chi-1) \text {. }
$$

Making the substitution $\eta=\frac{1}{2} \chi$, the eigenvalue equation

$$
W_{1 \mathrm{ink}} \psi(\eta)=\omega_{l} \psi(\eta)
$$

becomes

$$
\left[\frac{d^{2}}{d \eta^{2}}+32 x \kappa^{2}(\cos \eta-1)+4 \omega_{l}\right] \psi(n)=0
$$

which is Mathieu's equation. In later calculations we will take advantage of the fact that the eigenvalues $\omega_{l}$ have been computed and tabulated. ${ }^{20}$ 


\section{PERTURBATION THEORY AND THE VACUUM}

In order to perturb around $x=0$, we use our unperturbed Hamiltonian

$$
W_{0}=\sum_{n}\left[L^{2}(n)+\frac{x}{\kappa^{2}} \pi^{2}(n)+8 x \kappa^{2}\right],
$$

our perturbation then being

$$
\begin{aligned}
4 x V=-4 x \sum_{n}\left[\phi^{\dagger}(n) U(n, n+1) \phi(n+1)\right. \\
\left.+ \text { H.c. }-2 \kappa^{2}\right] .
\end{aligned}
$$

The zeroth-order ground state is then clearly a state for which

$$
L^{2}(n)|0\rangle=0 .
$$

Considering the first-order contribution from $W_{0}$, we see that the $\phi$ dependence of the ground state is

$$
\pi^{2}(n)|0\rangle=0 \text {. }
$$

We then note that

$$
\begin{aligned}
& L^{2}(n)[U(n, n+1)]^{m}|0\rangle=m^{2}[U(n, n+1)]^{m}|0\rangle, \\
& \pi^{2}(n)[\phi(n)]^{m}|0\rangle=m^{2}[\phi(n)]^{m}|0\rangle,
\end{aligned}
$$

enabling us to calculate the intermediate state energies required in the perturbation expansion. Since we include the diagonal $\left(x / \kappa^{2}\right) \pi^{2}$ in the unperturbed Hamiltonian, we only have even orders in the perturbation theory for the ground state, odd orders being obtained by expanding the $x$-dependent energy denominators.

Thus the zeroth-order energy of the vacuum state is 0 , the first order is $8 x \kappa^{2} N$. The second order is given by the graph of Fig. 3 . The perturbation creates a single $\phi$ excitation on one site and an anti- $\phi$ excitation on an adjacent site, joined by an electric flux string $U$. Clearly on an $N$ site (link) lattice this can be done in $2 N$ ways. The amplitude for this "creation" is $-4 x \kappa^{2}$. The second perturbation annihilates this excitation to return us to the initial state. The energy denominator is clearly

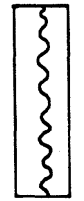

FIG. 3. Second-order graph contributing to the vacuum energy.

$$
\omega_{0}^{(0)}-\omega_{0}^{\prime}=-\left(1+\frac{2 x}{\kappa^{2}}\right)
$$

using Eq. (4.1a). Thus the total second-order contribution is

$$
-\frac{2\left(4 x \kappa^{2}\right)^{2} N}{\left(1+2 x / \kappa^{2}\right)}
$$

The fourth-order contribution is given by the graphs in Fig. 4 and their various time orderings. Fig. 4(a) represents the contribution where excitations are produced on different, nonadjacent links and later annihilated. This yields a total contribution

$$
-\frac{\left(4 x \kappa^{2}\right)^{4} N(N-3) \times 2 \times 4}{2\left(1+2 x / \kappa^{2}\right)^{3}} .
$$

Figures 4(b) and 4(c) give the contributions where the perturbation is applied on adjacent links. In Fig. 4(b) both excitations are created with a $\phi\left(\phi^{\dagger}\right)$ on the common site, while in Fig. 4(c) one excitation is created with a $\phi$ and the other a $\phi^{\dagger}$ on the common link. The contributions are

$$
-\frac{8 N}{\left[1+2\left(x / \kappa^{2}\right)\right]^{2}\left[2+6\left(x / \kappa^{2}\right)\right]}\left(4 x \kappa^{2}\right)^{4}
$$

and

$$
-\frac{8 N}{\left[1+2\left(x / \kappa^{2}\right)\right]^{2}\left[2+2\left(x / \kappa^{2}\right)\right]}\left(4 x \kappa^{2}\right)^{4},
$$

respectively. Finally, in Fig. 4(d) we have the case where the two excitations are "created" (then annihilated) on the same link, giving

$$
-\frac{2 N}{\left[1+2\left(x / \kappa^{2}\right)\right]^{2}\left[4+8\left(x / \kappa^{2}\right)\right]}\left(4 x \kappa^{2}\right)^{4} \text {. }
$$

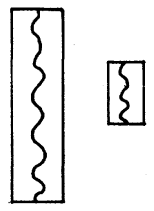

(a)

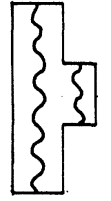

(c)

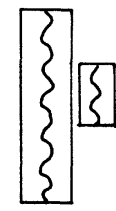

(b)



(d)
FIG. 4. Fourth-order graphs contributing to the vacuum energy. 
In addition, at fourth order we have the term of the Rayleigh-Schrödinger theory, ${ }^{19}$

$$
\begin{array}{r}
-\left(4 x \kappa^{2}\right)^{4}\left\langle 0\left|V \frac{1}{\omega_{0}^{(0)}-W_{0}} V\right| 0\right\rangle\left\langle 0\left|V \frac{1}{\left(\omega_{0}^{(0)}-W_{0}\right)^{2}} V\right| 0\right\rangle \\
=\frac{\left(4 x \kappa^{2}\right)^{4} N^{2} 4}{\left(1+2 x / \kappa^{2}\right)^{3}},
\end{array}
$$

which just cancels the $N^{2}$ contribution leaving the required extensive result.

This calculation can easily be extended to sixth order. The eighth order can then be trivially obtained using the following observation.

If we are terminating our series at eighth order, we can set $x$ to zero in the energy denominators of our eighth-order calculation. This is equivalent to calculating our eighth-order graphs using a Hamiltonian with $\pi^{2}(n) \equiv 0$. This problem has already been solved in the preceding section, since it reduces to solving Mathieu's equation on each link. We thus need the eighth-order term of $N \omega_{0} \imath$ where $\omega_{0 l}$ is the appropriate ground-state energy $\left(a_{0}\right)$ of the Mathieu equation. From Ref. 20, p. 724 , we find

$$
\omega_{0 l}=\frac{1}{4} a_{0}\left(16 x \kappa^{2}\right) .
$$

Thus the contribution of the eighth-order graphs is

$$
\frac{68687.4^{7}}{18874368} N\left(4 x \kappa^{2}\right)^{8}
$$

Such methods were used for the highest-order graphs for all the states we have calculated.

After expanding in powers of $x$ we end up with the following power series expansion for $\omega_{0}$, the $\omega$ energy of the vacuum,

$$
\begin{aligned}
\omega_{0}= & 2(4 x)-2(4 x)^{2}+(4 x)^{3}+3(4 x)^{4}-5(4 x)^{5} \\
& -\frac{595}{72}(4 x)^{6}+\frac{4199}{144}(4 x)^{7}+\frac{107911}{5760}(4 x)^{8}+\cdots,
\end{aligned}
$$

for $\kappa=1$, for example.

We can learn a great deal about this model if we generalize this calculation to the energy density of the ground state of the theory formulated in a background electric field $\alpha$. As discussed in Ref. 13 we can implement this idea by placing widely separated equal and opposite (fractional) charges into the system. Then a calculation of the groundstate energy density gives us the interquark potential which has already been discussed for $\kappa^{2}>1 / \pi$ in the Euclidean formulation of the theory. Note that the versions of the theory with different $\alpha\left(|\alpha|<\frac{1}{2}\right)$ cannot mix with each other through the perturbation $V$. At zeroth order in $x$ these states have the properties

$$
\begin{aligned}
& L(n)|\alpha\rangle=\alpha|\alpha\rangle, \\
& \pi(n)|\alpha\rangle=0,
\end{aligned}
$$

for all links and sites. The first statement of Eq. (4.11) states that the fractional charge $\alpha$ is accompanied by a flux tube of the correct strength to satisfy Gauss's law.

Before turning to the actual calculation it is interesting to interpret the parameter $\alpha$ in the decoupled $\left[\pi^{2}(n)=0\right]$ Mathieu theory. There $\alpha$ labels a state in the lowest band for which $\alpha=0$ is the lowest-lying state. ${ }^{20}$ If we replace the Mathieu equation by the harmonic oscillator and call the lattice spacing $1, \alpha$ corresponds to choosing a lattice with origin $\alpha$ rather than zero. In this case it is clear that $\alpha$ cannot effect the continuum limit of the theory since it just corresponds to a slightly different lattice formulation of a potential model. In other words the entire $\alpha<\frac{1}{2}$ band must become degenerate with the $\alpha=0$ state in the continuum limit (a well-known property of the Mathieu equation), i.e., the interquark potential vanishes in the decoupled theory in the limit $x \rightarrow \infty$. Of course, the full lattice field theory will not be so simple.

A perturbative calculation of the $\omega$ energy of an " $\alpha$ vacuum" involves the same graphs as that for the "true" vacuum. The zeroth-order $\omega$ energy is

$$
\omega_{0}^{(0)}=\alpha^{2} N,
$$

while the first order is simply

$$
\omega_{\alpha}^{(1)}=8 x \kappa^{2} N .
$$

The second-order contribution is given by the graph of Fig. 3 and is,

$$
-\left[\frac{1}{\left(1+2 \alpha+2 x / \kappa^{2}\right)}+\frac{1}{\left(1-2 \alpha+2 x / \kappa^{2}\right)}\right]\left(4 x^{2} \kappa^{4}\right) N .
$$

A graphical calculation including sixth order can then be supplemented by a single link (Mathieu) calculation to obtain the series for $\omega_{\alpha}$ through eighth order.

Now we can extract the physics from these calculations. The computation of $\omega_{\alpha}$ produces a series,

$$
\omega_{\alpha}=N S_{\alpha},
$$

where $S_{\alpha}$ is a dimensionless Taylor series in $x$ which is independent of $N$, the number of links of the lattice. The real energy of the $\alpha$ vacuum above the $\alpha=0$ vacuum is

$$
E_{\alpha}-E_{0}=\frac{a g^{2}}{2}\left(\omega_{\alpha}-\omega_{0}\right)=g \frac{a g}{2} N\left(S_{\alpha}-S_{0}\right),
$$


where we have used Eq. (3.8) relating $W$ to $H$. The number of links making up the lattice is $L / a$ where $L$ is the length of the system in physical units, say. Therefore,

$$
\frac{1}{L}\left(E_{\alpha}-E_{0}\right)=\frac{g^{2}}{2}\left(S_{\alpha}-S_{0}\right) \text {. }
$$

We shall call $\left(E_{\alpha}-E_{0}\right) / L$ the "string tension" $\tau_{\alpha}$ and compute

$$
\tau_{\alpha} /\left(\frac{1}{2} g^{2}\right)=S_{\alpha}-S_{0} \text {. }
$$

Now it is clear how to compute $\tau_{\alpha} /\left(\frac{1}{2} g^{2}\right)$ from the series for $S_{\alpha}$-form diagonal Padé approximants for $S_{\alpha}-S_{0}$ and take the continuum limit $x \rightarrow \infty$ directly. This is the same method used in other lattice theories ${ }^{3,19}$ to take the continuum limit. In particular, dimensionless quantities were identified (such as ratio of masses or dimensionless matrix elements) and the strong-coupling expansions were extrapolated to the continuum limit using diagonal Padé approximants as in analogous statistical-mechanics calculations. ${ }^{21}$

Using the expansions for $S_{\alpha}-S_{0}$ which we have calculated to eighth order in $x$ for arbitrary $\alpha$ and $\kappa^{2}$, we form the $[4,4]$ Padé approximants and take the continuum limits. In Table I we show the results for $\left(1 / \alpha^{2}\right)\left(S_{\alpha}-S_{0}\right)$ as a function of $\kappa^{2}$ for $\alpha$ $=0.3$. The division by $\alpha^{2}$ is done to measure the string tension relative to the strong-coupling ( $x$ $=0$ ) value of $\left(1 / \alpha^{2}\right)\left(S_{\alpha}-S_{0}\right)=1$. (See Tables II and III.) The results in Table III are also plotted in Fig. 5. Similar results are obtained for all values of $\alpha,|\alpha|<\frac{1}{2}$. We learn from this curve that the string tension persists in the continuum limit for $\kappa^{2}<\kappa_{c}^{2} \approx 0.7$ but vanishes for $\kappa^{2}>\kappa_{c}{ }^{2}$. So, our sus picion that this theory confines in the continuum limit for $\kappa^{2}$ small enough appears very possible. The fact that the string tension is slightly negative for $\kappa^{2}>\kappa_{c}{ }^{2}$ should not (we think) be taken seriously. The tension is within $7 \%$ of zero for $\kappa^{2}>\kappa_{c}^{2}$ so within the context of an approximate eighth-order calculation this result is compatible with zero, the expected answer for large $\kappa^{2}$.

Since the Hamiltonian and Euclidean formulations of the model use different cutoff procedures, the parameter $\kappa$ maps in a nontrivial fashion from one formulation to the other. Our estimate $\kappa_{c}{ }^{2} \approx 0.6-0.8$ applies to the Hamiltonian formulation and $\kappa_{c}{ }^{2} \approx 1 / \pi$ applies to the Euclidean formulation. (The reader should bare this in mind later in the text where we do not make this distinction explicitly.)

\section{SIMPLE MESONIC STATES}

To understand this lattice theory and its continuum limit in more detail, it is necessary to study its low-lying mass spectrum. In the strong-cou-

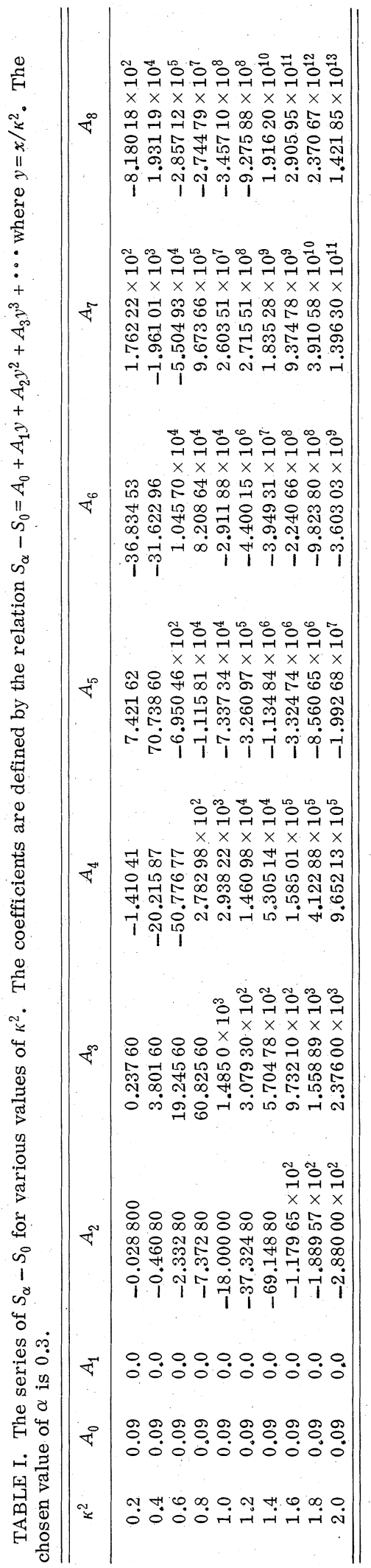




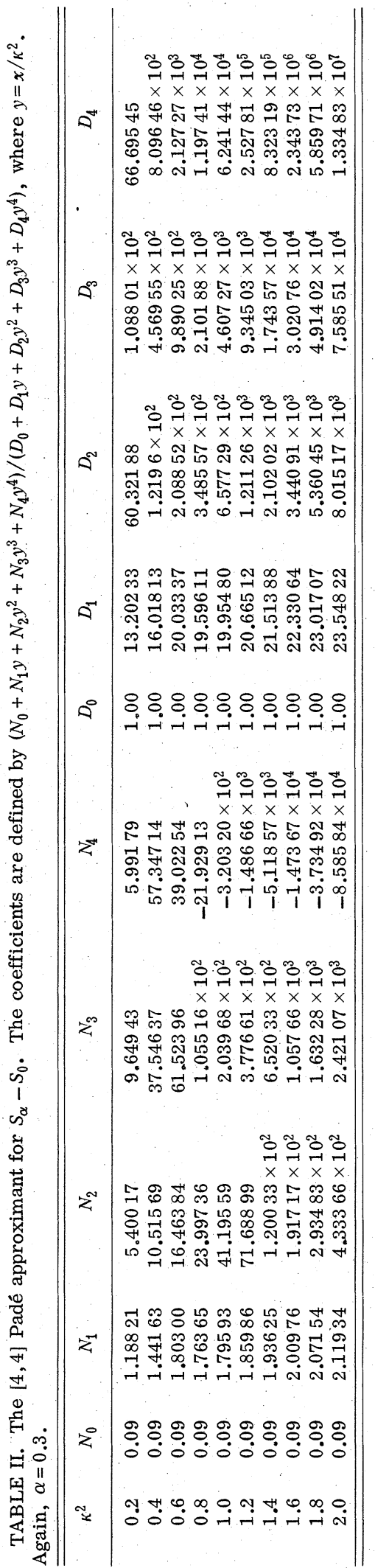

TABLE III. Values of the string tension, $\tau_{\alpha} /\left(\frac{1}{2} g^{2}\right)$, divided by the strong-coupling limit for various values of $\kappa^{2}$. The right-hand column is obtained from the Padé approximants of Table II in the continuum limit, $x \rightarrow \infty$.

\begin{tabular}{cc}
\hline$\kappa^{2}$ & $\frac{1}{\alpha^{2}}\left(S_{\alpha}-S_{0}\right)$ \\
\hline 0.2 & 0.998 \\
0.4 & 0.787 \\
0.6 & 0.204 \\
0.8 & -0.020 \\
1.0 & -0.057 \\
1.2 & -0.065 \\
1.4 & -0.068 \\
1.6 & -0.070 \\
1.8 & -0.071 \\
2.0 & -0.071 \\
\hline \hline
\end{tabular}

pling limit ( $x$ small) simple gauge-invariant states are made by applying $\phi(n)$ to one site, $\phi^{\dagger}(n+1)$ to a nearest neighbor and connecting them by a flux line $U$. There are two such states whose zeromomentum unperturbed forms are

$\frac{1}{\sqrt{2 N}} \sum_{n}\left[\phi^{\dagger}(n) U(n, n+1) \phi(n+1)+\right.$ H.c. $]|0\rangle$

and

$\frac{1}{\sqrt{2 N} i} \sum_{n}\left[\phi^{\dagger}(n) U(n, n+1) \phi(n+1)-\right.$ H.c. $]|0\rangle$.

It is easy to see that in the naive continuum limit the latter state becomes a single massive vector meson $B_{1}\left[B_{\mu} \equiv A_{\mu}+(1 / g) \partial_{\mu} \theta\right]$ for which $m_{V} / g=\sqrt{2} \kappa$. The former (symmetric) state is identified with the unbound scalar state of mass $2 m_{V}$. In this respect these states are analogous to the low-lying vector and (unbound) scalar states of the massless Schwinger model. ${ }^{19}$

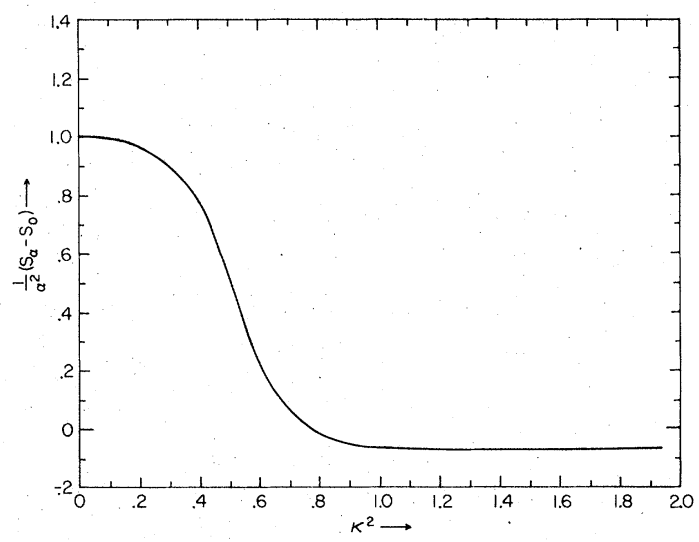

FIG. 5. String tension, $\tau_{\alpha} /\left(\frac{1}{2} g^{2}\right)$, for background field $\alpha=0.3$ plotted againt $\kappa^{2}$. 
To calculate the masses of the states in Eq. (5.1) perturbatively, it is convenient to deal with the states

$$
|+\rangle=\frac{1}{\sqrt{N}} \sum_{n} \phi^{\dagger}(n) U(n, n+1) \phi(n+1)|0\rangle
$$

and

$$
|-\rangle=\frac{1}{\sqrt{N}} \sum_{n} \phi^{\dagger}(n+1) U^{\dagger}(n, n+1) \phi(n)|0\rangle
$$

and consider a $2 \times 2$ mass matrix. We will label the diagonal $(++)$ matrix element of the RaleighSchrödinger perturbation theory $A$ and the mixing $(+-)$ between the states $B$. The energy of the vector (scalar) state is then $A-B(A+B)$.

To zeroth order such states have $\omega$ energies $1+2 x / \kappa^{2}$. The graphs contributing second-order terms to $A$ are listed in Fig. 6. The $\omega$ energy of each intermediate state is just the sum of the squares of the fluxes on each link plus $x / \kappa^{2}$ times the sum of the squares of the number of $\phi$ excitations on sites. The energy denominator is then $1+2 x / \kappa^{2}$ minus this $\omega$ energy for each graph of Fig. 6. We have for each graph

$$
\begin{aligned}
& (\mathrm{a})=-\frac{2(N-3)}{\left(1+2 x / \kappa^{2}\right)}\left(4 \kappa^{2} x\right)^{2}, \\
& (\mathrm{~b})=-\frac{2}{\left(1+4 x / \kappa^{2}\right)}\left(4 \kappa^{2} x\right)^{2}, \\
& (\mathrm{c})=-2\left(4 \kappa^{2} x\right)^{2}, \\
& (\mathrm{~d})=-\frac{1}{\left(3+6 x / \kappa^{2}\right)}\left(4 \kappa^{2} x\right)^{2}, \\
& (\mathrm{e})=\frac{N}{\left(1+2 x / \kappa^{2}\right)}\left(4 \kappa^{2} x\right)^{2}, \\
& (\mathrm{f})=-\frac{(N-3)}{\left(1+2 x / \kappa^{2}\right)}\left(4 \kappa^{2} x\right)^{2}, \\
& (\mathrm{~g})=-2\left(4 \kappa^{2} x\right)^{2} .
\end{aligned}
$$

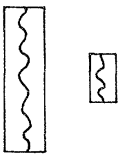

(a)

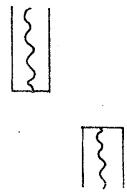

(e)

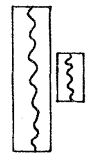

(b)

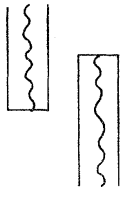

(f)

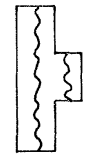

(c)

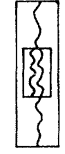

(d)
FIG, 6, Graphs contributing to the amplitude $A$ in second order,
The graphs contributing to $B$ at second order are shown in Fig. 7. Their values are

$$
\begin{aligned}
& (\mathrm{a})=\frac{N}{\left(1+2 x / \kappa^{2}\right)}\left(4 \kappa^{2} x\right)^{2}, \\
& (\mathrm{~b})=-\frac{(N-3)}{\left(1+2 x / \kappa^{2}\right)}\left(4 \kappa^{2} x\right)^{2}, \\
& (\mathrm{c})=-\frac{2}{\left(1+4 x / \kappa^{2}\right)}\left(4 \kappa^{2} x\right)^{2} .
\end{aligned}
$$

The calculation of the amplitudes $A$ and $B$ have been done by computer to sixth order. The eighthorder coefficient is obtained by using Mathieu's equation [one needs the characteristic roots labeled $b_{2}$ (vector) and $a_{2}$ (scalar)]. The series for the $\omega$ energies are given in Tables IV and V, while the $[4,4]$ Padé approximants are given in Tables VI and VII. We note that all the Padé approximants are free of zeros or poles for positive $x$. This is an important result because it indicates that none of the series have a tendency to vanish for finite $x$. In other words, the theory's mass gap (in units of the lattice spacing) does not appear to vanish for finite $x$. Thus, we find no evidence for any continuous phase transitions for finite $x$. However, we do find qualitatively different numerical values for the Padé approximants above and below $\kappa^{2}=\kappa_{c}{ }^{2}$ $\approx 0.6-0.8$. In particular consider the ratio of the vector-state mass at various $\kappa^{2}, m_{V}\left(\kappa^{2}\right)$, to the vector-state mass at $\kappa^{2}=2$, well above the suspected critical point. If we compute this ratio by taking the ratio of the $[4,4]$ Padé approximants for the corresponding $\omega$ energies and then let $x$ $\rightarrow \infty$, Table VIII results. Table VIII also contains a column showing

$$
\frac{m_{V}\left(\kappa^{2}\right)}{m_{V}\left(\kappa^{2}=2\right)}\left(\frac{2}{\kappa^{2}}\right)^{1 / 2} \text { vS } \kappa \text {. }
$$

This particular quantity is chosen because if the lattice theory had precisely the naive continuum limit for all $\kappa^{2}$, then $m_{V}\left(\kappa^{2}\right)=\sqrt{2} g \kappa$ and the quantity $\left[m_{V}\left(\kappa^{2}\right) / m_{V}(2)\right]\left(2 / \kappa^{2}\right)^{1 / 2}$ would be unity. This table and Fig. 8 indicate that the relationship $m_{V}\left(\kappa^{2}\right)=\sqrt{2} \kappa g$ is fairly well obeyed above $\kappa_{c}{ }^{2}$ $\approx 0.6-0.8$, but the vector-state mass becomes huge below the critical point.

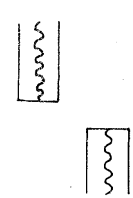

(a)

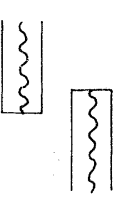

(b)

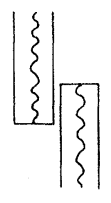

(c)
FIG. 7. Graphs contributing to the amplitude $B$ in second order. 

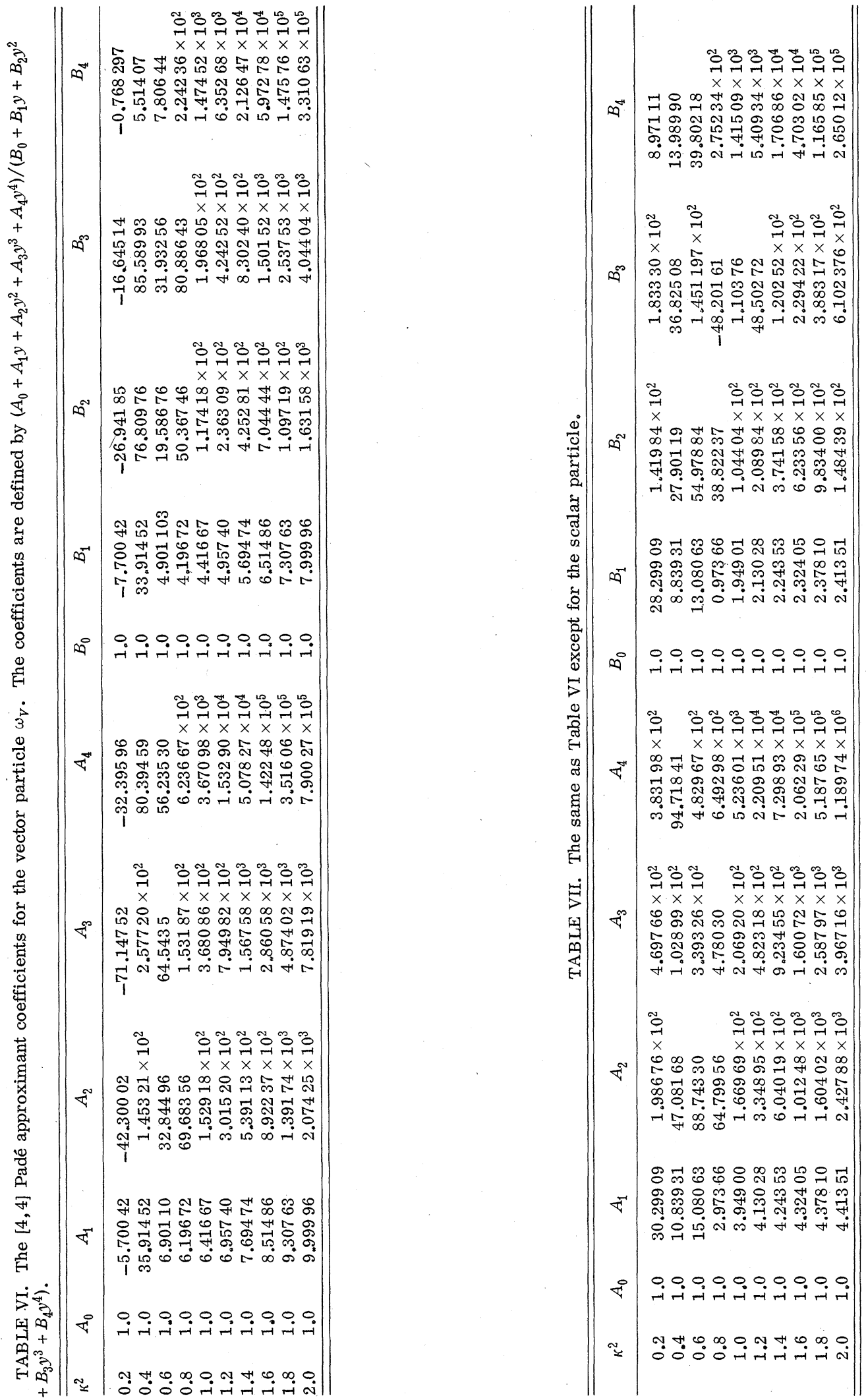
TABLE VIII. The continuum limit of the mass of the vector state at various $\kappa^{2}$ compared to its value at $\kappa^{2}$ $=2 \mathrm{vs}$ the parameter $\kappa^{2}$.

\begin{tabular}{ccc}
\hline$\kappa^{2}$ & $m_{V}\left(\kappa^{2}\right) / m_{V}(2)$ & $\left(\frac{2}{\kappa^{2}}\right)^{1 / 2} \frac{m_{V}\left(\kappa^{2}\right)}{m_{V}(2)}$ \\
\hline 0.2 & 17.66 & 55.84 \\
0.4 & 6.10 & 13.64 \\
0.6 & 3.01 & 5.50 \\
0.8 & 1.16 & 1.83 \\
1.0 & 1.04 & 1.47 \\
1.2 & 1.00 & 1.29 \\
1.4 & 1.00 & 1.20 \\
1.6 & 1.00 & 1.19 \\
1.8 & 1.00 & 1.05 \\
2.0 & 1.00 & 1.00 \\
\hline \hline
\end{tabular}

It is also informative to study the mass of the scalar state as a function of $\kappa^{2}$ in precisely the same way as we studied the vector. The corresponding table for the scalar mass $m_{s}\left(\kappa^{2}\right)$ and a plot of $m_{s}\left(\kappa^{2}\right) / m_{s}(2)$ vs $\kappa^{2}$ are given in Table IX and Fig. 9, respectively. These results indicate that the scalar particle becomes massless at the critical point. This means that the phase transition at $\kappa_{c}^{2}$ is continuous. In addition, the scalar particle, like the vector particle, becomes extremely massive in the small $\kappa^{2}$ phase.

One can also study the mass ratio $m_{s} / m_{V}$ as a function of $\kappa^{2}$. The most reliable procedure to employ in calculating this ratio in the continuum limit is to note that

$$
\frac{m_{s}}{m_{V}}=\frac{\omega_{s}}{\omega_{V}}
$$

is a dimensionless quantity which should be a finite real number. So, using the strong-coupling expansions for $\omega_{S}$ and $\omega_{V}$, we obtain the Taylor series to eighth order for $\omega_{S} / \omega_{V}$ by division and continue it to $x=\infty$ using a diagonal $[4,4]$ Padé

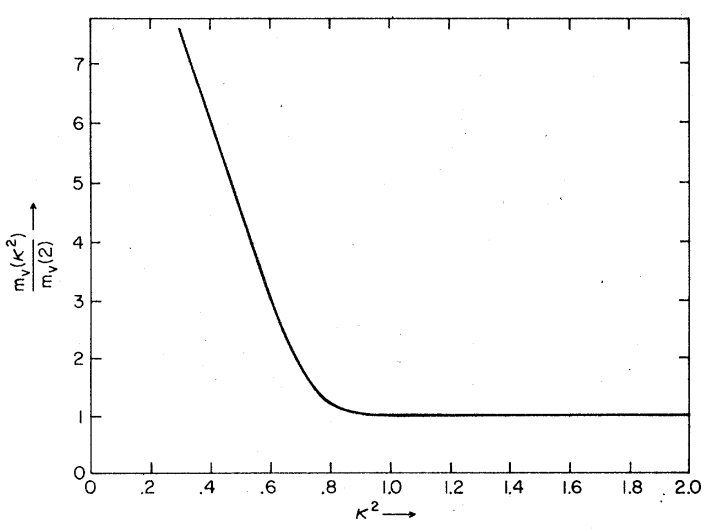

FIG. 8. Plot of the ratio of the $[4,4]$ Pade approximants for $m_{V}\left(\kappa^{2}\right)$ and $m_{V}\left(\kappa^{2}=2\right)$ vs $\kappa^{2}$.
TABLE IX. The same as Table VIII except for the scalar particle.

\begin{tabular}{lcc}
\hline \hline$\kappa^{2}$ & $\frac{m_{S}\left(\kappa^{2}\right)}{m_{S}(2)}$ & $\frac{m_{S}\left(\kappa^{2}\right)}{m_{S}(2)}\left(\frac{2}{\kappa^{2}}\right)^{1 / 2}$ \\
\hline 0.2 & 9.52 & 30.10 \\
0.4 & 1.51 & 3.38 \\
0.6 & 2.70 & 4.93 \\
0.75 & 0.26 & 0.42 \\
0.8 & 0.53 & 0.84 \\
1.0 & 0.82 & 1.16 \\
1.2 & 0.91 & 1.17 \\
1.4 & 0.95 & 1.14 \\
1.6 & 0.98 & 1.10 \\
1.8 & 0.99 & 1.04 \\
2.0 & 1.00 & 1.00 \\
\hline \hline
\end{tabular}

approximant. The results of this computation are given in Table $X$ and plotted in Fig. 10. They indicate, in agreement with the analysis above, that the scalar state becomes massless at $\kappa_{c}{ }^{2}$. How ever, one must be wary of this calculation since we have seen in Figs. 8 and 9 that the series for both $\omega_{S}$ and $\omega_{V}$ change their character as $\kappa^{2}$ passes through $\kappa_{c}{ }^{2}$. Therefore, taking the ratio of the individual series and forming a Padé approximant might be quite sensitive to the "noise" in each series. In fact, the series for $\omega_{s} / \omega_{V}$ and the Padé approximant itself are quite irregular just below $\kappa_{c}^{2}$.

Figure 10 also indicates that in the large $-\kappa^{2}$ phase,

$$
\frac{m_{S}}{m_{V}} \cong 1.84 \quad\left(\kappa^{2}>5\right) .
$$

This ratio lies $8 \%$ below the value 2.00 expected in a naive continuum limit. This is satisfactory agreement, but it is not a particularly profound test of our calculational method. The reason is that for large $\kappa^{2}$ the momentum conjugate to $\theta(n)$ enters the Hamiltonian in the expression $\pi^{2}(n) / \kappa^{2}$.

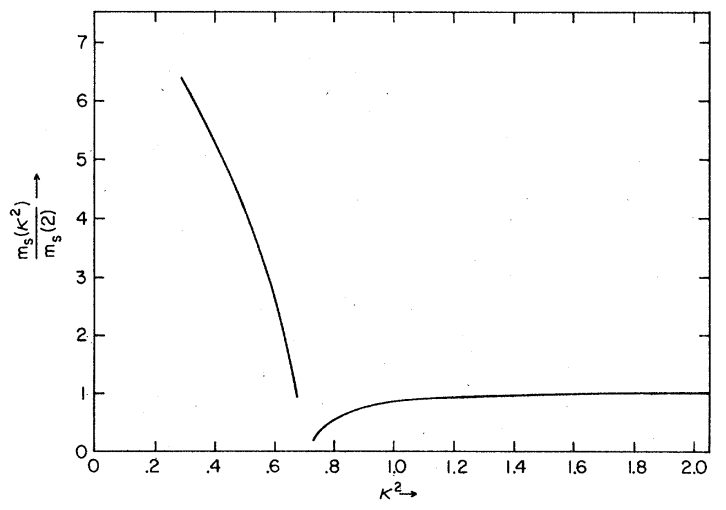

FIG. 9. Same as Fig. 8 except for the scalar particle. 
TABLE X. Continuum limit of the $[4,4]$ Pade approximant for $m_{S} / m_{V}=\omega_{S} / \omega_{V}$ as a function of $\kappa^{2}$. The ratios are quite irregular (and presumably unreliable) in the immediate vicinity of $\kappa_{c}^{2} \simeq 0.75-0.85$.

\begin{tabular}{cccc}
\hline \hline$\kappa^{2}$ & $m_{S} / m_{V}$ & $\kappa^{2}$ & $m_{S} / m_{V}$ \\
\hline 0.05 & 1.0001 & 0.60 & 2.037 \\
0.10 & 1.002 & 0.65 & 2.316 \\
0.15 & 1.008 & 0.70 & 2.802 \\
0.20 & 1.025 & 0.75 & 4.429 \\
0.25 & 1.060 & 0.80 & $\ldots .$. \\
0.30 & 1.120 & 0.85 & 0.272 \\
0.35 & 1.209 & 0.90 & 0.961 \\
0.40 & 1.329 & 0.95 & 1.238 \\
0.45 & 1.474 & 1.00 & 1.390 \\
0.50 & 1.640 & 2.00 & 1.807 \\
0.55 & 1.825 & 4.00 & 1.834 \\
& & 6.00 & 1.836 \\
& & $\cdots$ & $\cdots$ \\
\hline \hline
\end{tabular}

Hence, the quantum character of $\theta(n)$ becomes numerically insignificant for large $\kappa^{2}$ and the calculation of masses becomes essentially identical to the calculation of the level structure of the harmonic oscillator. (This observation was also made in Sec. III.) Nonetheless, Eq. (5.5) is quite satisfactory, although the possibility exists that the Padé results for $m_{S} / m_{V}$ will not converge monotonically to 2.00 since convergence is known to be quite irregular for potential models. ${ }^{22}$

Now it is interesting to abstract the phase diagram of the electrodynamics of the planar model from these calculations. For $\kappa^{2}>\kappa_{c}^{2} \approx 0.6-0.8$ the continuum limit of the theory exhibits the Higgs mechanism and for $\kappa^{2}$ well above $\kappa_{c}{ }^{2}$ it is well approximated by a free massive vector meson. How ever, for $\kappa^{2}<\kappa_{c}{ }^{2}$, the continuum limit of the theory appears to be the free, massless electromagnetic field. This last fact should be so because for $\kappa^{2}$ $<\kappa_{c}{ }^{2}$ the theory confines in the continuum limit and

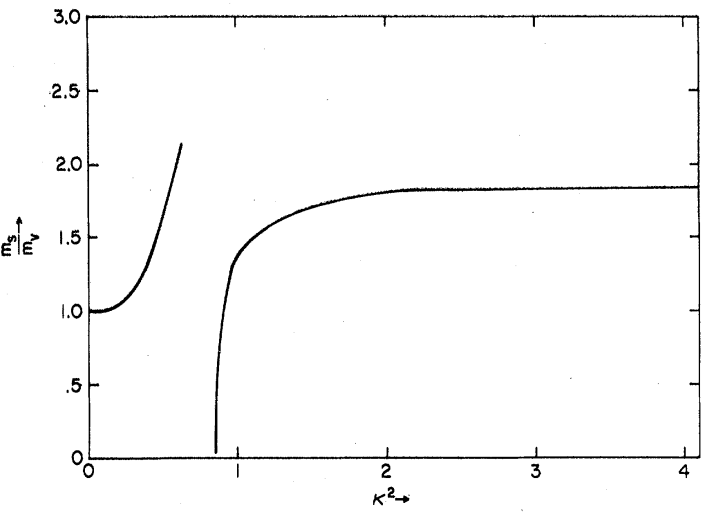

FIG. 10. Plot of the $[4,4]$ Padé approximant for the ratio $m_{S} / m_{V}$ vs $\kappa^{2}$.

has no finite mass spectrum. In other words, we are interpreting the graphs of Figs. 8 and 9 to mean that the vector and scalar mesons move to infinite mass, leaving behind the free electromagnetic field. Presumably if higher orders were calculated $m_{V}\left(\kappa^{2}\right) / m_{V}(2)$ would steadily increase for $\kappa^{2}<\kappa_{c}^{2}$. We feel that this is a reasonable interpretation of Figs. 8 and 9, since the plots change so dramatically as $\kappa^{2}$ passes through $\kappa_{c}{ }^{2}$.

There are other intuitive reasons for suspecting this result. From the perspective of Sec. II, below $\kappa_{c}^{2}$ the vortices become dense in the continuum limit and can be expected to remain relevant. They then preserve the confining property of the theory as $a \rightarrow 0$. In addition, they disorder the theory on the length scale of the lattice spacing itself. The size of the vortices, $g$, becomes unimportant since they overlap. Therefore, it is reasonable to expect the masses of the theory to be $O(1 / a)$ or precisely zero. Hence, the vector and scalar states disappear as $a \rightarrow 0$, and a massless confining potential remains behind.

\section{THE EUCLIDEAN FORMULATION REVISITED}

It is interesting to return to the Euclidean formulation and search for the phases of the model in light of the results found in Secs. IV and V. Consider Eq. (2.26)

$$
Z(J)=\int_{-\infty}^{\infty} \prod_{r} d \phi(r) \sum_{m(r)=-\infty}^{\infty} \exp \left[-\frac{1}{4 \kappa^{2}} \sum_{r}\left(\Delta_{\nu} \phi\right)^{2}-\frac{g^{2} a^{2}}{2} \sum_{r}\left(\phi+\frac{e}{g} J\right)^{2}+2 \pi i \sum_{r} m(r) \phi(r)\right] .
$$

Following the strategy used to understand the planar model, ${ }^{16}$ we generalize this expression to one which contains an adjustable activation energy of an isolated bare vortex,

$$
Z(J, A) \equiv \int_{-\infty}^{\infty} \prod_{r} d \phi(r) \sum_{m(r)=-\infty}^{\infty} \exp \left[-\frac{1}{4 \kappa^{2}} \sum_{r}\left(\Delta_{\nu} \phi\right)^{2}-\frac{g^{2} a^{2}}{2} \sum_{r}\left(\phi+\frac{e}{g} J\right)^{2}-A \sum_{r} m(r)^{2}+2 \pi i \sum_{r} m(r) \phi(r)\right] .
$$

It is interesting to suppose that $A \gg 1$, so the sum over $m(r)$ can be well approximated by keeping just the 
$m(r)=0, \pm 1$ terms. Then,

$$
\begin{aligned}
\sum_{m(r)=-\infty}^{\infty} e^{-A m(r)^{2}+2 \pi i m(r) \phi(r)} & \approx 1+2 e^{-A} \cos [2 \pi \phi(r)] \\
& \approx e^{2 e^{-A} \cos [2 \pi \phi(r)]} .
\end{aligned}
$$

So, in this approximation,

$$
Z(J, A) \approx \int_{-\infty}^{\infty} \prod_{r} d \phi(r) \exp \left[-\frac{1}{4 \kappa^{2}} \sum_{r}\left(\Delta_{\nu} \phi\right)^{2}-\frac{g^{2} a^{2}}{2} \sum_{r}\left(\phi+\frac{e}{g} J\right)^{2}+2 e^{-A} \sum_{r} \cos [2 \pi \phi(r)]\right] .
$$

It is best to rescale $\phi(r) \rightarrow \phi(r) / \sqrt{2} \kappa$ and replace sums over lattice sites by integrals over space-time,

$$
Z(J, \mu) \simeq \int_{-\infty}^{\infty} \prod_{r} d \phi(r) \exp \left\{-\int d^{2} x\left[\frac{1}{2}\left(\partial_{\nu} \phi\right)^{2}+\frac{2 \kappa^{2} g^{2}}{2}\left(\phi+\frac{e}{\sqrt{2} \kappa g} J\right)^{2}-\mu \cos (2 \pi \sqrt{2} \kappa \phi]\right\}\right.
$$

where $\mu=2 e^{-A} / a^{2}$. Setting $J=0$, we see that our generalized model is equivalent to the lattice sine-Gordon theory with a mass term $\frac{1}{2} m_{V}{ }^{2} \phi^{2}$, where $m_{V}=\sqrt{2} \kappa g$. In addition, for $\kappa>1 / \sqrt{\pi}$ this model is known to be equivalent to the electrodynamics of the Thirring model. ${ }^{23}$ Observe also that at $\kappa=1 / \sqrt{\pi}$ the argument of the cosine term becomes $\sqrt{8 \pi} \phi$ - this is the special point where the ground state of the continuum model falls to minus infinity. ${ }^{24}$ For. $\kappa=1 / \sqrt{2 \pi}$ the argument of the cos term becomes $\sqrt{4 \pi}$ and the theory reduces to the massive Schwinger model [the fermion mass $\sim$ the parameter $\mu$ in Eq. (6.4)].

Of course, what we really need to know is the quantity $\mu$ itself. In recent studies of the pure planar model the renormalization-group trajectories in the $(\mu, \kappa)$ plane were determined. ${ }^{12,16}$ In this way one related the theory at one point $\left(\mu_{1}, \kappa_{1}\right)$ to the theory at another $\left(\mu_{2}, \kappa_{2}\right)$. Then the introduction of the parameter $\mu$ could be justified as just a helpful device. These studies found the fixed line of spin wave theory and the KosterlitzThouless phase transition was studied in quantitative detail. ${ }^{12,16}$

A detailed renormalization-group analysis of this model can be done and will be reported elsewhere. Here we will only present a crude "twostep" renormalization which is adequate for $\kappa^{2}$ $>1 / \pi$ and a sketch of the general approach. Return to Eq. (2.29),

$$
\begin{aligned}
Z J J=0)=\sum_{m(r)} \exp \left(-4 \pi^{2} \kappa^{2} \sum_{r, r^{\prime}}\right. & m(r) \\
& \left.\times G\left(r-r^{\prime} ; m_{V} a\right) m\left(r^{\prime}\right)\right) .
\end{aligned}
$$

Recall that if interactions between vortices are neglected, one obtains Eq. (2.31),

$$
Z(J=0) \approx \sum_{m(r)} \exp \left(2 \pi \kappa^{2} \ln \left(m_{V} a\right) \sum_{r} m(r)^{2}\right) .
$$

So, the activation energy for an isolated vortex is

$$
\tilde{A}=-2 \pi \kappa^{2} \ln \left(m_{V} a\right) .
$$

We have seen in Sec. II that for $m_{V} a$ sufficiently small and $\kappa^{2}$ sufficiently large, the gas of vortices is dilute and Eq. (2.31) is a good approximation to the real system. These observations suggest the following two-step renormalization procedure appropriate for $\kappa^{2}>1 / \pi$.

Consider Eq. (6.1) again and organize the integration over $\phi(r)$ into a "high" momentum slice and a "low" momentum slice. Include in the "high" momentum slice all the frequency components of $\phi$ greater than $\left(m_{V}^{\prime} a\right)^{-1}$ and include all other momenta in the "low" momentum slice. Let $\bar{\phi}$ denote the field with the high-momentum components removed. Then explicitly integ rating the high-momentum components of $\phi(r)$ out of Eq. (2.26) gives

$$
\begin{aligned}
Z(J)=\int_{-\infty}^{\infty} \prod_{r} d \bar{\phi}(r) \sum_{m(r)=-\infty}^{\infty} \exp [ & -\frac{1}{4 \kappa^{2}} \sum_{r}\left(\Delta_{\nu} \bar{\phi}\right)^{2}-\frac{g^{2} a^{2}}{2} \sum_{r}\left(\bar{\phi}+\frac{e}{g} J\right)^{2} \\
& \left.-4 \pi^{2} \kappa^{2} \sum_{r_{0} r^{\prime}} m(r) \tilde{G}\left(r-r^{\prime} ; m_{V} a\right) m\left(r^{\prime}\right)+2 \pi i \sum_{r} m(r) \bar{\phi}(r)\right],
\end{aligned}
$$


where $\tilde{G}$ is the Green's function for $\phi-\bar{\phi}$, the high-momentum components of $\phi(r)$. Let us replace $\tilde{G}\left(r-r^{\prime} ; m_{V} a\right)$ by $-\tilde{A} \delta_{r, r^{\prime}}$. As reviewed above, this approximation is good for $\kappa^{2}>1 / \pi$. Then the manipulations in Eq. (6.2) can be applied again giving

$$
\begin{aligned}
& Z(J)=\int_{-\infty}^{\infty} \prod_{r} d \bar{\phi}(r) \exp \left[-\frac{1}{4 \kappa^{2}} \sum_{r}\left(\Delta_{\nu} \bar{\phi}\right)^{2}-\frac{g^{2} a^{2}}{2} \sum_{r}\left(\bar{\phi}+\frac{e}{g} J\right)^{2}+2 e^{-\tilde{A}} \sum_{r} \cos (2 \pi \bar{\phi}(r))\right], \\
& Z(J)=\int_{-\infty}^{\infty} \prod_{r} d \bar{\phi}(r) \exp \left\{-\int d^{2} x\left[\frac{1}{2}\left(\partial_{\nu} \bar{\phi}\right)^{2}+\frac{2 \kappa^{2} g^{2}}{2}\left(\bar{\phi}+\frac{e}{\sqrt{2} \kappa g} J\right)^{2}-2 m_{V}{ }^{2}\left(m_{V} a\right)^{2\left(\pi \kappa^{2-1}\right)} \cos (2 \pi \sqrt{2} \kappa \bar{\phi}]\right\} .\right.
\end{aligned}
$$

Now we can let $a \rightarrow 0$. For $\kappa^{2}>1 / \pi$, the coefficient of the cosine term vanishes in the continuum limit and we have

$$
Z(J)-\int_{-\infty}^{\infty} \prod_{r} d \bar{\phi}(r) \exp \left\{-\int d^{2} x\left[\frac{1}{2}\left(\partial_{\nu} \bar{\phi}\right)^{2}+\frac{2 \kappa^{2} g^{2}}{2}\left(\bar{\phi}+\frac{e}{2 \sqrt{\kappa} g} J\right)^{2}\right]\right\},
$$

which the reader can identify as the massless Schwinger model. This theory exhibits the Higgs mechanism and does not confine-by translating $\bar{\phi}$, the external source $J$ is eliminated from the expression for the free energy. In addition, the mass of the boson $\bar{\phi}$ is $m_{V}=\sqrt{2} \kappa g$ in agreement with our earlier analyses.

For general $\kappa^{2}$ such a naive two-step renormalization must be replaced by an infinite sequence of integrations. Consider Eq. (6.3) after restoring continuum space-time notation,

$$
Z(J, \mu)=\int_{-\infty}^{\infty} \prod_{r} d \phi(r) \exp \left[\int d^{2} x \frac{1}{4 \kappa^{2}}\left(\partial_{\nu} \phi\right)^{2}+\frac{g^{2}}{2}\left(\phi+\frac{e}{g} J\right)^{2}-\mu \cos [2 \pi \phi(r)]\right],
$$

where $\kappa^{2}$ and $\mu$ are considered to be bare parameters of the theory. The lattice provides an ultraviolet cutoff on the theory, the largest permissible Euclidean momentum is $p<1 / a$. Now consider integrating in a sequence of infinitesimal steps all the momenta in the theory between $1 / a$ and a finite value on the order of, but considerably larger than $1 / g$. Then in each of these steps the superrenormalizable mass term $\frac{1}{2} g^{2}[\phi+(e / g) J]^{2}$ in Eq. (6.9) is irrelevant and can be ignored. Therefore, the infinite renormalizations in the model of Eq. (6.9) are the same as those of the ordinary sineGordon theory. But this is (essentially) the model studied by Kosterlitz, ${ }^{12}$ so we can apply his analysis directly. In particular, for $\kappa^{2}<1 / \pi$, his work suggested that the $\left(\kappa^{2}, \mu\right)$ trajectories flow to a fixed point where both $\kappa^{-2}$ and $\mu$ are large (presumably infinite). In other words, the equivalent temperature of the theory formulated on a coarse lattice is infinite and the ground state consists of a condensate of vortices. If the coefficient of the cosine term in Eq. (6.9) becomes large, then $\phi$ will freeze at values where $\cos [2 \pi \phi(r)]=1$. In addition, if the coefficient of the gradient term in Eq. (6.9) becomes large, $\phi(r)$ will become a con- stant. Requiring that the partition function be nontrivial, then picks out the value zero for $\phi(r)$. The Wilson loop integral becomes

$$
Z(J) / Z(0)-\exp \left(-\frac{1}{2} e^{2} \int J^{2}(r) d^{2} x\right)
$$

which implies an interquark potential of

$$
E(R)=\frac{1}{2} e^{2} R \text {. }
$$

So, an unscreened electric flux tube extends between the quarks in agreement with the strongcoupling expansions for $\kappa^{2}<\kappa_{c}^{2}$.

In summary, this renormalization-group-analysis and the results of strong-coupling expansions suggest that there is a fixed line of theories for $\kappa^{2}>\kappa_{c}{ }^{2}$ which exhibit the Higgs mechanism, that the fixed line terminates and that for $\kappa^{2}<\kappa_{c}{ }^{2}$ the renormalization-group trajectories flow to a $\kappa^{2}$ $=0$ fixed point which is the free electromagnetic field.

\section{EXCITED STATES (LOCAL EXCITATIONS)}

Naively, following our experience with the Schwinger model ${ }^{19}$ we would expect that local ex- 
cited states could only be created from the vacuum by successive applications of the gauge-invariant operators $\phi^{\dagger}(n) U(n, n+1) \phi(n+1)$ and its Hermitian conjugate. Actually there are a far larger number of gauge-invariant operators allowed in this theory since there clearly exists a sensible definition of the operator $\left[\phi^{\dagger}(n) U(n, n+1) \phi(n+1)\right]^{\alpha}$ and its Hermitian conjugate for any real $\alpha$.

Hence we will consider those states ohtained by applying $\left[\phi^{\dagger}(n) U(n, n+1) \phi(n+1)\right]^{\alpha}$ to the vacuum, or their zero-momentum analogs obtained by summing over $n$. We will first consider the case in which $\alpha \neq \frac{1}{2} n$ ( $n$ an integer) and also not equal to certain other values, determined later, for which a degenerate perturbation theory calculation is necessary.

The zeroth- and first-order contributions are clearly

$$
\omega_{\alpha}^{(0)}=\alpha^{2}\left(1+\frac{2 x}{\kappa^{2}}\right)
$$

after vacuum subtraction. The second-order contribution to the $\omega$ energy is clearly given by the graphs of Fig. 11 where the dashed lines represent the fractionally charged $\phi^{\alpha}$ 's and their associated fluxes $U^{\alpha}$. In each of these graphs, the perturbation creates a scalar-antiscalar particle pair with its associated flux $U\left(U^{\dagger}\right)$ and then annihilates it on the same link. Figure 11(a) is the contribution where the vacuum excitation is not adjacent to the initial excited link, and contributes

$$
\frac{-(N-3) 2}{\left(1+2 x / \kappa^{2}\right)}\left(4 x \kappa^{2}\right)^{2} \text {. }
$$

In Fig. 11(b), the perturbation excites a link adjacent to the initial excited link yielding

$$
-2\left\{\frac{1}{\left[1+(2+2 \alpha) x / \kappa^{2}\right]}+\frac{1}{\left[1+(2-2 \alpha) x / \kappa^{2}\right]}\right\}\left(4 \kappa^{2} x\right)^{2} .
$$

Finally we have the case shown in Fig. 11(c) where the perturbation acts on the excited link changing its flux to $(\alpha \pm 1)$ giving

$$
-\left[\frac{1}{(1+2 \alpha)}+\frac{1}{(1-2 \alpha)}\right] \frac{1}{\left(1+2 x / \kappa^{2}\right)}\left(4 \kappa^{2} x\right)^{2} .
$$

This calculation has been extended to sixth order in $x$, (see Fig. 12) and by use of the single-link

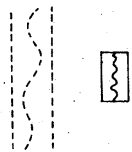

(a)

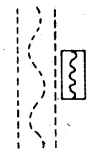

(b)

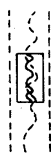

(c)
FIG. 11. Second-order graphs contributing to the $\omega$ energy of an $\alpha$ excitation.

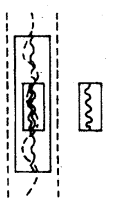

(a)

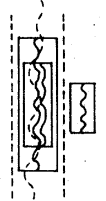

(b)

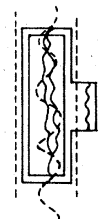

(c)
FIG. 12. Sixth-order graphs contributing to the $\omega$ energy of an $\alpha$ excitation.

(Mathieu) calculation, to eighth order.

The quantity $m_{\alpha} / g=\omega_{\alpha} / \sqrt{8 x}$ has been calculated for a range of values of $\alpha$ and $\kappa^{2}$, and it has been plotted as a function of $x$ in Figs. 13(a) and 13(b) for $\kappa^{2}=1$. In order to dispose of the factor $\sqrt{8 x}$ which prevents us from taking the limit $x \rightarrow \infty$ directly, we have considered the ratio $m_{\alpha} / m_{0.75}$, expanded it as a power series in $x$ to eighth order and expressed it as a $[4,4]$ Padé approximant for a range of values of $\kappa^{2}$. The results appear in Table XI and are plotted in Figs. 14(a) and 14(b). Before discussing these results we must mention a peculiar property of these states: An $\alpha$ excita-
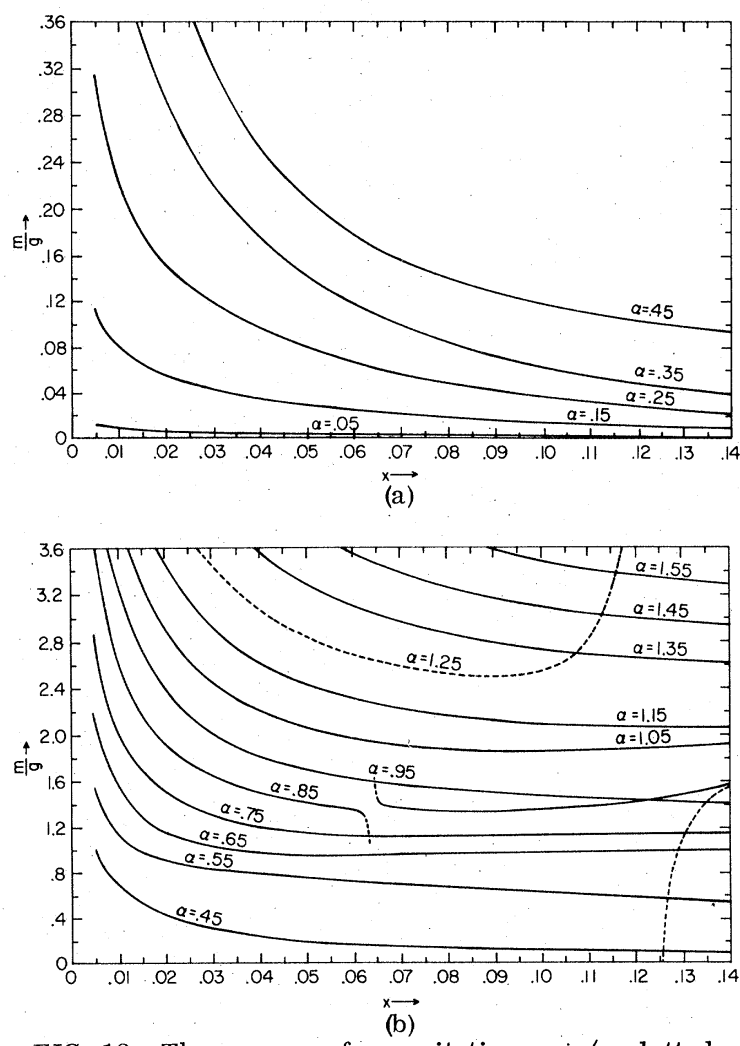

FIG. 13. The masses of $\alpha$ excitations $m_{\alpha} / g$ plotted vs $\kappa^{2}$ for $\alpha$ between 0.05 and 0.45 in (a); and between 0.45 and 1.55 in (b). 
TABLE XI. Continuum limit of the [4,4] Padé approximant calculation of $m_{\alpha} / m_{0.75}$ as a function of $\kappa^{2}$ and $\alpha$.

\begin{tabular}{|c|c|c|c|c|c|c|c|}
\hline$\alpha k^{2}$ & 0.2 & 0.4 & 0.6 & 0.8 & 1.0 & 1.4 & 1.8 \\
\hline 0.05 & 0.00415 & 0.00140 & -0.00019 & -0.00064 & -0.00083 & -0.00095 & -0.00098 \\
\hline 0.15 & 0.03726 & 0.01203 & -0.00188 & -0.00661 & -0.00934 & -0.01159 & -0.01223 \\
\hline 0.25 & 0.10273 & 0.02999 & -0.00529 & -0.01870 & -0.02797 & -0.03662 & -0.03927 \\
\hline 0.35 & 0.35169 & 0.05010 & -0.00779 & -0.02064 & -0.02436 & -0.02630 & -0.02674 \\
\hline 0.45 & 0.28775 & 0.12961 & 0.09773 & 0.09146 & 0.08970 & 0.08878 & 0.08857 \\
\hline 0.55 & 0.61129 & 0.75927 & 0.78915 & 0.79505 & 0.79671 & 0.79757 & 0.79776 \\
\hline 0.65 & 0.75196 & 0,90750 & 0.94822 & 0.95886 & 0.96205 & 0.96374 & 0.96412 \\
\hline 0.75 & 1.00000 & 1.00000 & 1.00000 & 1.00000 & 1.00000 & 1.00000 & 1.00000 \\
\hline 0.85 & 1.27655 & 1.23992 & -2.74160 & 0.94846 & 0.98000 & 0.99049 & 0.99245 \\
\hline 0.95 & 1.58740 & 1.43786 & 1.35442 & 1.71833 & -0.74246 & 0.62147 & 0.69570 \\
\hline $1-$ & 2.06004 & 0.52988 & 0.01221 & 1.5785 & 1.08027 & 0.96476 & 0.94446 \\
\hline $1+$ & 1.85780 & -0.32929 & 1.27693 & 1.38573 & 1.40169 & 1.40312 & 1.40240 \\
\hline 1.05 & 1.93426 & 1.57833 & 0.95384 & 1.24105 & 1.27722 & 1.28799 & 1.28976 \\
\hline 1.15 & 2.31496 & 1.69225 & 1.54487 & 1.45485 & 1.42384 & 1.40623 & 1.40211 \\
\hline 1.25 & 2.69686 & 2.12989 & 1.74143 & 1.62513 & 1.58867 & 1.56904 & 1.56458 \\
\hline
\end{tabular}

tion on one link cannot be annihilated by the perturbation and recreated on another link. Therefore, these states have no momentum dependence. We call such states which cannot move "arthritic."

Let us first consider those states for which $|\alpha|$ $<\frac{1}{2}$. They have the property that the perturbation does not mix them with states of lower (zeroth order) energy. The corresponding band of states of the decoupled mode Hamiltonian (Mathieu equa-

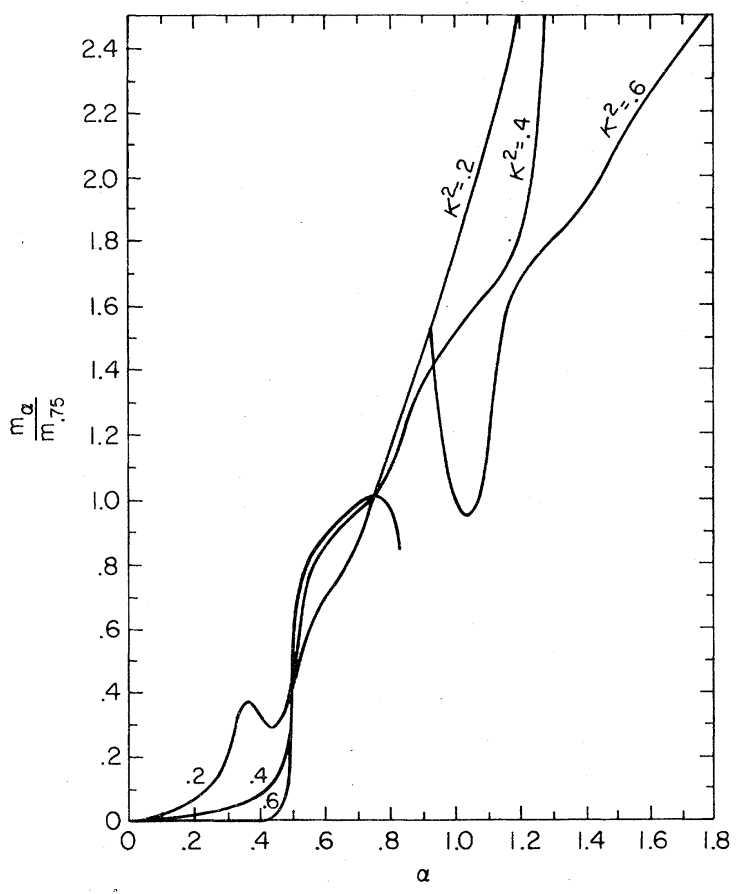

(a) tion $)^{25}$ attain zero energy as $x-\infty$. From Fig. 14(b) we see that they do indeed form a separate band of zero mass for $\kappa^{2} \geqslant \kappa_{c}^{2} \approx 0.6$. We believe that the small (less than 10\%) negative values of $m_{\alpha} / m_{0,75}$ just represent limitations of $[4,4]$ Padé approximants in approximating zero. Below $\kappa_{c}{ }^{2}$ we expect the confining property of the continuum limit to cause $m_{\alpha} / m_{0.75}$ to be a monotonic function of $\alpha$ for $|\alpha|<\frac{1}{2}$. This is, in fact, what we learn

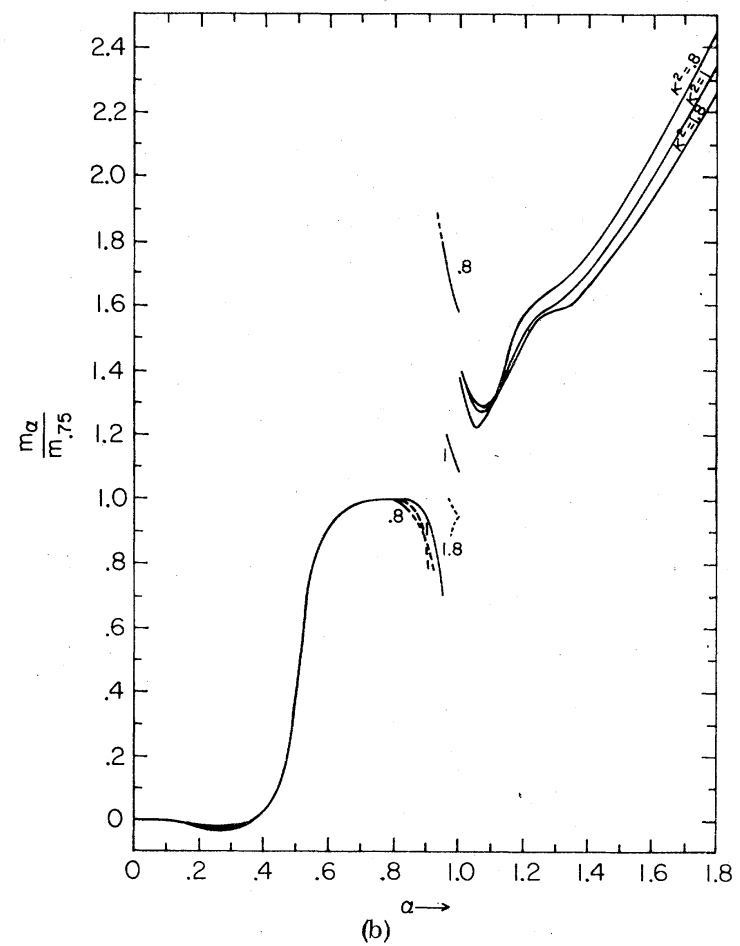

FIG. 14. Plot of the $[4,4]$ Padé approximant to $m_{\alpha} / m_{0} .75$ vs $\alpha$ for $\kappa^{2}$ between 0.2 and 0.6 in (a); and between 0.8 and 1.8 in (b). 
from Table XI and Fig. 14(a).

In summary, the $|\alpha|<\frac{1}{2}$ band of states is degenerate with the vacuum in the continuum limit for $\kappa^{2}>\kappa_{c}{ }^{2}$. As discussed in Sec. IV the $\alpha$ vacuums become degenerate in the continuum limit for $\kappa^{2}>\kappa_{c}{ }^{2}$ because of screening (the Higgs mechanism). Clearly the local, arthritic states (for $|\alpha|<\frac{1}{2}$ ) become degenerate with the vacuum because of the same mechanism.

It is clear that the arthritic states considered here are only a small subset of all such vacuum excitations. All gauge-invariant states for which the flux on each link is less than $\frac{1}{2}$ fall into this category. That they are stable, despite the fact that their energies above the vacuum can be arbitrarily large (for a sufficiently coarse lattice) is a consequence of the inability of the confining theory to screen nonintegral charge.

Let us now move to the consideration of the next band, $\frac{1}{2}<|\alpha|<1$, its boundaries defined by a zeroth-order degeneracy and its associated level repulsion. The decoupled Hamiltonian ${ }^{25}$ leads us to associate this band with the first excited state, the vector particle, of the continuum limit for $\kappa^{2}$ $>\kappa_{c}{ }^{2}$. We see from Table $X$ that the $[4,4]$ Padé approximants for the mass ratios $m_{\alpha} / m_{0.75}$ lie within $20 \%$ of the expected value 1 independent of $\alpha$ for $0.6<\alpha<1$. Although we could have hoped for better numerical values, the fact that the $\frac{1}{2}<|\alpha|<1$ is qualitatively distinct from the vacuumlike $0<|\alpha|<\frac{1}{2}$ band is certainly clear from Fig. 14(b) where a plateau exists for $0.6<\alpha<1$. In addition we have calculated the antisymmetric $\alpha=1$ state's mass to eighth order and find $m_{1}^{(-)} / m_{0.75}=1.080271$ which falls within $9 \%$ of unity. For $\kappa^{2}<\kappa_{c}{ }^{2}$ the $\frac{1}{2}<.|\alpha|<1$ band does not become degenerate in the continuum limit.

Thus, considering mass alone, our interpretation of the band $\frac{1}{2}<|\alpha|<1$ as being a set of states which become degenerate representations of the vector particle of mass $\sqrt{2} \kappa g$ seems good (for $\kappa^{2}$ $>\kappa_{c}{ }^{2} \approx 0.6$, of course). However, this interpretation poses a conceptual problem. For the uppermost state of the band $(\alpha=1)$ the single-particle interpretation is clear since the excitation can be moved by the perturbation from one site to another. The other states of the band are, however, arthritic, so how can one interpret them as singleparticle states?

To shed light on this, consider a link having flux $\beta$ and suppose that $-\frac{1}{2}<\beta<0$. Consider now the action of $\phi^{\dagger}(n) U(n, n+1) \phi(n+1)$ or its Hermitian conjugate on each link of the lattice. If it were applied uniformly to the $\alpha=0$ vacuum state, we would obtain the vector or scalar state with $\alpha$ $= \pm 1$. It has a zeroth-order $\omega$ energy of 1 . In the case at hand, if the operator is applied on any link other than the excited one we get an excitation with flux \pm 1 on this link, while leaving flux $\beta$ on the excited link. An $\omega$ energy $1+\beta^{2}>1$ ensues. If, however, the operator acts on the excited link it produces a flux $1+\beta=\alpha$ and an $\omega$ energy $\alpha^{2}<1$. Its Hermitian conjugate produces a flux $-1+\beta$ and an $\omega$ energy $(1-\beta)^{2}>+\beta^{2}$. The interpretation is now clear. The vacuum excitation $\beta$ acts as a scattering center (defect) situated on one link of the lattice. For small $x$ there exists one bound state with zeroth-order $\omega$ energy $\alpha^{2}$, and scattering states with zeroth-order $\omega$ energy $1+\beta^{2}$ and $(1-\beta)^{2}$ indicating a potential which is attractive or repulsive, depending on the parity of the incoming particle. The spectrum of scattering states only appears strange because momentum dependence of the scattering state energies only appears at order $x^{2}$ when the higher momentum scalar states can feel the repulsion and connect with the state of $\omega$ energy $(1-\beta)^{2}$. Now we see why our states of flux $\frac{1}{2}<|\alpha|<1$ are arthritic: It is simply because the vector particle is bound to the vacuum excitation $\beta$ which is itself arthritic. As $x \rightarrow \infty$, the vacuum excitation becomes a genuine vacuum and the particle unbinds, which is the reason that this state becomes a representative of the vector particle state in the continuum limit.

It is more difficult to identify the third and higher-lying bands in Fig. 14(b). There is an indication of a band at $m_{\alpha} / m_{0_{0} 75} \approx 1.6-1.7$ which we would like to identify with the scalar particle (the precise ratio should be 2). Apparently our eighth-order calculation is not powerful enough to adequately describe the continuum limit of higher-lying bands.

Finally we note that new degeneracies not present in the decoupled theory occur causing higher bands to break up into sub-bands, presumably a prelude to their becoming continuum rather than discrete states when $a=0$. The first such new boundary is at $\alpha=\frac{5}{4}$. In general they occur at $\alpha$ $=\frac{1}{2}(n+n / m)$, where $n$ and $m$ are integers.

\section{THE VACUUM IN BACKGROUND FIELD $\frac{1}{2}$}

The problem of the $\alpha$ vacuums becomes more difficult when $\alpha=\frac{1}{2}$, since the perturbation connects the state with all fluxes $\frac{1}{2}$ to states where some fluxes are $-\frac{1}{2}$. To zeroth order these states are degenerate with the initial state. Moreover, a state with a single charged $\phi$ (or $\phi^{\dagger}$ ) excitation on 1 site can have fluxes $\frac{1}{2}$ on one side, $-\frac{1}{2}$ on the other, a state which is again degenerate with the vacuum at zeroth order. Thus we see a phenomenon first noticed in the massive Schwinger model and true for the lattice Schwinger model even for zero mass ${ }^{7}$ : Charged particles are unconfined for a vacuum field $\frac{1}{2}$. 
In order to explore this problem more fully it is expedient to write down an effective Hamiltonian whose eigenstates solve the zeroth-order degeneracy problem. The Hamiltonian is

$$
\begin{gathered}
W=\sum_{n}\left\{L^{2}(n)+x \kappa^{-2} \pi^{2}(n)\right. \\
-4 x\left[\phi^{\dagger}(n) U(n, n+1) \phi(n+1)\right. \\
\left.\left.+ \text { H.c. }-2 \kappa^{2}\right]\right\} .
\end{gathered}
$$

For the states of interest $|\alpha|=\frac{1}{2}$ on each link. Thus

$$
\sum_{n} L^{2}(n)=\frac{1}{4} N
$$

and can be dropped. There are only two allowed states for each link $\alpha=\frac{1}{2}$ or $\alpha=-\frac{1}{2}$. The operators $\phi^{\dagger}(n) U(n, n+1) \phi(n+1)$ and its Hermitian conjugate simply take us from one of these states to the other. We therefore define a spin variable which is $+\frac{1}{2}$ for $\alpha=\frac{1}{2}$ and $-\frac{1}{2}$ for $\alpha=-\frac{1}{2}$. The $\phi^{\dagger}(n) U(n, n+1) \phi(n+1)$ and its Hermitian conjugate are just $\kappa^{2}$ times the spin raising and lowering operators $\sigma_{+}(n), \sigma_{-}(n)$. As for $\pi^{2}(n)$, it yields 0 if the two links adjacent the $n$th site have the same flux, and 1 if one has $\alpha=\frac{1}{2}$, the other $\alpha=-\frac{1}{2}$. Thus we can write it in terms of spin variables as

$$
\pi^{2}(n)=\frac{1}{2}\left[1-\sigma_{z}(n) \sigma_{z}(n+1)\right] .
$$

Thus, removing an irrelevant constant

$$
W=-\frac{1}{2} x \kappa^{-2} \sum_{n}\left[\sigma_{\varepsilon}(n) \sigma_{\varepsilon}(n+1)+8 \kappa^{4} \sigma_{x}(n)\right],
$$

which is the Hamiltonian for the one-dimensional Ising model in a transverse magnetic field. It is a soluble model ${ }^{26}$ and has the following two phases:

A. $8 \kappa^{4}<1$. The system is magnetized, i.e., the ground-state expectation value of $\sigma_{z}$ is nonvanishing.

B. $8 \kappa^{4}>1$. The system is disordered and the ground state consists of a kink condensate. ${ }^{27}$ In terms of the disorder parameter,

$$
\mu_{z}(m)=\prod_{n>m} \sigma_{x}(n)
$$

the ground state satisfies $\left\langle 0\left|\mu_{z}(m)\right| 0\right\rangle \neq 0$. These two phases are separated by a second-order phase transition.

The physical meaning of the phases $A$ and $B$ considered in terms of the original variables $\phi$ and $U$ is as follows. For $8 \kappa^{4}>1$, the ground state is a kink condensate. But the kink operator $\mu_{z}(m)$ is just a phase operator $\exp [i \phi(m)]$ with a string of flux extending to the right. This is so because

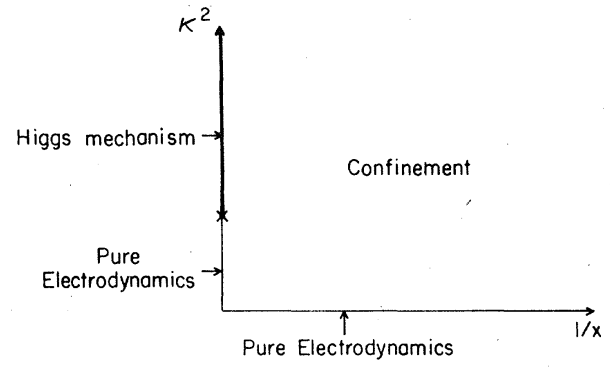

FIG. 15. The phase diagram of the electrodynamics of the planar model found in this approximate study.

$$
\sigma_{x}(n)-\phi^{\dagger}(n) U(n, n+1) \phi(n+1)+\text { H.c. }
$$

and from Eq. (8.4),

$$
\mu_{z}(m) \longrightarrow \phi^{\dagger}(m) \prod_{n>0} U(m, m+n)
$$

So, the disordered phase of the Ising model corresponds to a condensation of gauge-invariant matter fields in the original model, i.e., the Higgs mechanism. So, in this phase confinement is surely lost even in the lattice theory $(a \neq 0)$. For $8 \kappa^{4}<1$ the expectation value of the operator $\mu_{z}(m)$ vanishes in the ground state and the theory confines for strong coupling.

\section{CLOSING REMARKS}

Rather than summarize all the calculations and detailed conclusions occurring in this study, we will just remind the reader of the points we feel are most interesting.

According to our analysis the phase diagram of this model lattice gauge theory is that shown in Fig. 15. For fixed finite $x$ and any $\kappa^{2}$, the theory confines. In the continuum limit and $\kappa^{2}>\kappa_{c}{ }^{2} \approx 0.6-$ 0.8 the theory displays the Higgs mechanism. The simple possibility that the theory is then just a free massive vector meson with $m_{V}=\sqrt{2} \kappa g$ is supported by several calculations. For $\kappa^{2}<\kappa_{c}{ }^{2}$ the

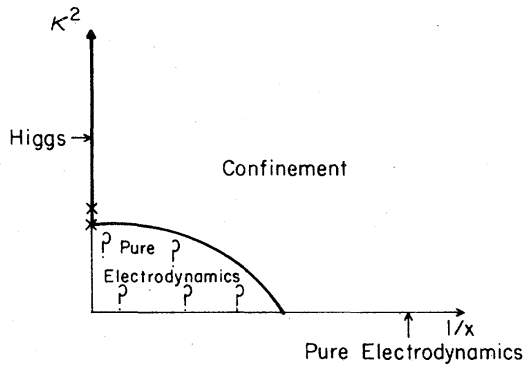

FIG. 16. A speculative phase diagram. "Pure electrodynamics" is a linear potential while "confinement" has a nontrivial mass spectrum. 
continuum limit appears to be just the free electromagnetic field. It confines and is simply a linear, static potential. We have argued that the existence of $\kappa_{c}^{2}$ is linked to the presence of vortices in the lattice gauge theory and that for $\kappa^{2}$ $>\kappa_{c}{ }^{2}$ they become infinitely dilute and irrelevant in the continuum limit while for $\kappa^{2}<\kappa_{c}{ }^{2}$ they do not.

Might the phase diagram really be more complicated than this? Since our calculations were only approximate, they could have missed some interesting structure. Since the lattice theory contains two-dimensional parameters $g$ and $a$, the critical region $\kappa^{2} \approx \kappa_{c}{ }^{2}$ could be more complicated than the simple ending of a fixed line. Another interesting possibility is that the pure electrodynamics phase really extends a finite distance into the phase diagram as shown in Fig. 16. We have searched for this possibility using our series expansions and found no evidence for it. We certainly, however, cannot rule it out.

The original motivation for studying this model was to gain some insight into a field theory which could lose confinement precisely at $a=0$. We have seen that Hamiltonian strong-coupling expansions were quite efficient and unambiguous in uncovering this fact. This gives us some confidence that if a similar "catastrophe" occurs in non-Abelian gauge theories in $3+1$ dimensions, it should show up plainly in our work.

\section{ACKNOWLEDGMENTS}

Parts of this work have been presented and discussed in seminars by one of us (J.K.). He thanks L. Susskind and his colleagues at SLAC for comments. Suggestions of S. Shenker played an important role in developing the Euclidean formulation of the model. One of us (D.K.S.) thanks the University of Illinois for support and hospitality during the last phase of this work. We thank our colleagues at the University of Illinois for constructive comments. D.K.S. thanks M. Stone and his colleagues at the University of Cambridge for helpful discussions.

This work was supported in part by the National Science Foundation under Grant No. NSF 77-2579.
${ }^{1}$ A. M. Polyakov, Phys. Lett. 59B, 82 (1975).

${ }^{2}$ K. G. Wilson, Phys. Rev. D 10,2445 (1974).

${ }^{3} \mathrm{~J}$. Kogut and L. Susskind, Phys. Rev. D 11, 395 (1975).

${ }^{4} \mathrm{G}$. 't Hooft, in Renormalization of Yang-Mills Fields and Applications to Particle Physics, edited by C. P. Korthals-Altes (C.N.R.S., Marseille, France, 1972); H. D. Politzer, Phys. Rev. Lett. $\underline{30}, 1346$ (1973); D. J. Gross and F. Wilczek, ibid. 30,1343 (1973).

${ }^{5} \mathrm{~J}$. Kogut, D. K. Sinclair, and L. Susskind, Nucl. Phys. B114, 199 (1976); T. Banks, D. R. T. Jones, J. Kogut, S. Raby, P. N. Scharbach, D. K. Sinclair, and L. Susskind, Phys. Rev。D 15, 1111 (1977).

${ }^{6}$ R. Savit and M. Einhorn, Phys. Rev. D 17, 2583 (1978); H. R. Quinn and M. Weinstein, Phys. Rev. D 17, 1063 (1978); R. B. Israel and C. R. Nappi, Harvard Univ. Report. No. HUTMP-78/B56, 1978 (unpublished).

${ }^{7}$ S。 Coleman, Ann. Phys. (N. Y。) 101, 239 (1976); D. R. T. Jones, J. B. Kogut, J. Shigemitsu, D. K. Sinclair, and P. N. Scharbach, report (unpublished).

${ }^{8}$ A. M. Polyakov, Nucl. Phys. B120, 429 (1977).

${ }^{9}$ H. E. Stanley and T. A. Kaplan, Phys. Rev. Lett. 17, 913 (1966).

${ }^{10}$ N. D. Mermin and H. Wagner, Phys. Rev. Lett. $\underline{17}$, 1133 (1966).

${ }^{11} \mathrm{~J} . \mathrm{M}$. Kosterlitz and D. J. Thouless, J. Phys. C $\underline{6}$, 1181 (1973)。

${ }^{12}$ J. M. Kosterlitz, J. Phys. C 7 , 1046 (1974).

${ }^{13}$ S. Coleman, R. Jackiw, and L. Susskind, Ann. Phys. (N. Y。) 93, 267 (1975).

${ }^{14} \mathrm{~J}$. Villain, J. Phys. (Paris) 36, 581 (1975).
${ }^{15}$ R. Savit, Phys. Rev. Lett. $\underline{39}, 55$ (1977).

${ }^{16}$ J. V. José, L. P. Kadanoff, S. Kirkpatrick, and D. R. Nelson, Phys. Rev. B 16, 1217 (1977).

${ }^{17}$ S. T. Chui and J. D. Weeks, Phys. Rev。 B 14, $4978^{\circ}$ (1976).

${ }^{18}$ C. G. Callan, R. Dashen, and D. J. Gross, Phys. Rev. D 16, 2526 (1977); S. Raby and A. Ukawa, ibid. 18, 1154 (1978).

${ }^{19} \mathrm{~T}$. Banks, L. Susskind, and J. Kogut, Phys. Rev. D 17 , 1043 (1976).

${ }^{20}$ Handbook of Mathematical Functions, edited by M. Abramowitz and I. A. Stegun (Dover, New York, (1968).

${ }^{21} \mathrm{~A}$ review of these methods is contained in J. B. Kogut in Many Degrees of Freedom in Field Theory, proceedings of the 8th International Summer Institute on Theoretical Physics, Bielefeld, W. Germany, 1976, edited by L. Streit (Plenum, N。Y., 1978).

${ }^{22}$ J. Jurkiewicz and J. Wosiek, Nucl. Phys. B135, 416 (1978).

${ }^{23}$ J. Kogut and D. K. Sinclair, Phys. Rev. D 1 0, 4181 (1974).

${ }^{24}$ S. Coleman, Phys. Rev. D 11, 2088 (1975).

${ }^{25}$ N. W. McLacklan, Theory and Application of Mathieu Functions (Clarendon, Oxford, England, 1947). The band structure can also be seen in Ref. 20, Fig. 20.6, p. 728.

${ }^{26}$ P. Pfeuty, Ann. Phys. (N. Y.) 57, 79 (1970).

${ }^{27}$ E. F radkin and L. Susskind, Phys. Rev. D 17, 2637 (1978). 